\title{
Tuning the Topology of Three-dimensional Covalent Organic Frameworks via Steric Control: From pts to Unprecedented ljh
}

\author{
Yang Xie, ${ }^{1}$ Jian Li, ${ }^{2,3}$ Cong Lin, ${ }^{2}$ Bo Gui, ${ }^{1}$ Chunqing Ji, ${ }^{4}$ Daqiang Yuan, ${ }^{4}$ \\ Junliang Sun ${ }^{2 *}$ and Cheng Wang ${ }^{1 *}$
}

${ }^{1}$ Sauvage Center for Molecular Sciences, College of Chemistry and Molecular Sciences, Wuhan University, Wuhan 430072, China

${ }^{2}$ College of Chemistry and Molecular Engineering, Beijing National Laboratory for Molecular Sciences, Peking University, Beijing 100871, China

${ }^{3}$ Department of Materials and Environmental Chemistry, Stockholm University, Stockholm 10691, Sweden

${ }^{4}$ State Key Laboratory of Structural Chemistry, Fujian Institute of Research on the Structure of Matter, Chinese Academy of Sciences, Fuzhou 350002, China

†These authors contributed equally to this work: Yang Xie, Jian Li

Email: chengwang@whu.edu.cn (C. W.) junliang.sun@pku.edu.cn (J. S.) 


\section{Section 1. Materials and Methods}

All reagents and solvents, unless otherwise noted, were purchased from commercial sources and used without further purification. Compound $\mathbf{1}^{\mathrm{S} 1}, \mathbf{2}^{\mathrm{S} 2}, \mathbf{4}^{\mathrm{S} 3}$ and tetra $(p-$ aminophenyl) methane (TAPM) ${ }^{\mathrm{S} 4}$ were synthesized from reported procedures.

${ }^{1} \mathrm{H}$ and ${ }^{13} \mathrm{C}$ NMR spectra were measured on a Bruker Fourier $400 \mathrm{M}$ spectrometer. High resolution mass spectra were collected on Bruker Solarix. Elemental analysis was conducted on a Flash EA 1112. Fourier transform infrared (FTIR) spectra were recorded on a Nicolet iN10 micro FTIR Spectrometer. Powder X-ray diffraction (PXRD) patterns were obtained on a Rigaku SmartLab X-Ray diffractometer with $\mathrm{Cu}$ $\mathrm{K} \alpha$ line focused radiation at $45 \mathrm{kV}$ and $200 \mathrm{~mA}$ or Rigaku MiniFlex $600 \mathrm{X}$-Ray diffractometer. In situ powder X-ray diffraction patterns were obtained on a Rigaku SmartLab SE with $\mathrm{Cu} K \alpha$ line focused radiation at $40 \mathrm{kV}$ and $50 \mathrm{~mA}$. The sample was put in a heating cell chamber and the diffraction patterns were acquired at a regular interval range from $30{ }^{\circ} \mathrm{C}$ to $500{ }^{\circ} \mathrm{C}$ with a heating rate of $10{ }^{\circ} \mathrm{C} \mathrm{min}-1$ under nitrogen flow. Thermogravimetric analysis (TGA) from $30-800{ }^{\circ} \mathrm{C}$ was carried out on a TAQ500 in nitrogen atmosphere using a $10^{\circ} \mathrm{C} / \mathrm{min}$ ramp without equilibration delay. Field-emission scanning electron microscope (FE-SEM) was performed on a ZEISS SIGMA operating at an accelerating voltage ranging from 0.1 to $20 \mathrm{kV}$.

The ${ }^{13} \mathrm{C}$ CP MAS spectra were recorded on a Bruker AVANCE NEO $400 \mathrm{WB}$ spectrometer equipped with a $4 \mathrm{~mm}$ standard bore. The CP MAS probe head whose X channel was tuned to $100.62 \mathrm{MHz}$ for ${ }^{13} \mathrm{C}$ and the other channel was tuned to 400.18 $\mathrm{MHz}$ for broad band ${ }^{1} \mathrm{H}$ decoupling, using a magnetic field of $9.39 \mathrm{~T}$ at $297 \mathrm{~K}$. The dried and finely powdered samples were packed in the $\mathrm{ZrO}_{2}$ rotor closed with Kel-F cap which were spun at $8 \mathrm{kHz}$ rate. The experiments were conducted at a contact time of $2 \mathrm{~ms}$. A total of 2000 scans were recorded with 3 s recycle delay for each sample. All ${ }^{13} \mathrm{C}$ CP MAS chemical shifts are referenced to the resonances of adamantane standard.

The nitrogen isotherms were measured at $77 \mathrm{~K}$ using an Autosorb-iQ (Quantachrome) surface area size analyzer. Before measurement, the samples were 
degassed in vacuum at $120{ }^{\circ} \mathrm{C}$ for $12 \mathrm{~h}$. Oil-free vacuum pumps and oil-free pressure regulators were used for measurements to prevent contamination of the samples during the degassing process and isotherm measurement. The Brunauer-Emmett-Teller (BET) method was utilized to calculate the specific surface areas. The pore size distributions were calculated by a nonlocal density functional theory (NLDFT).

\section{Section S2. Synthesis of precursors and 3D COFs}

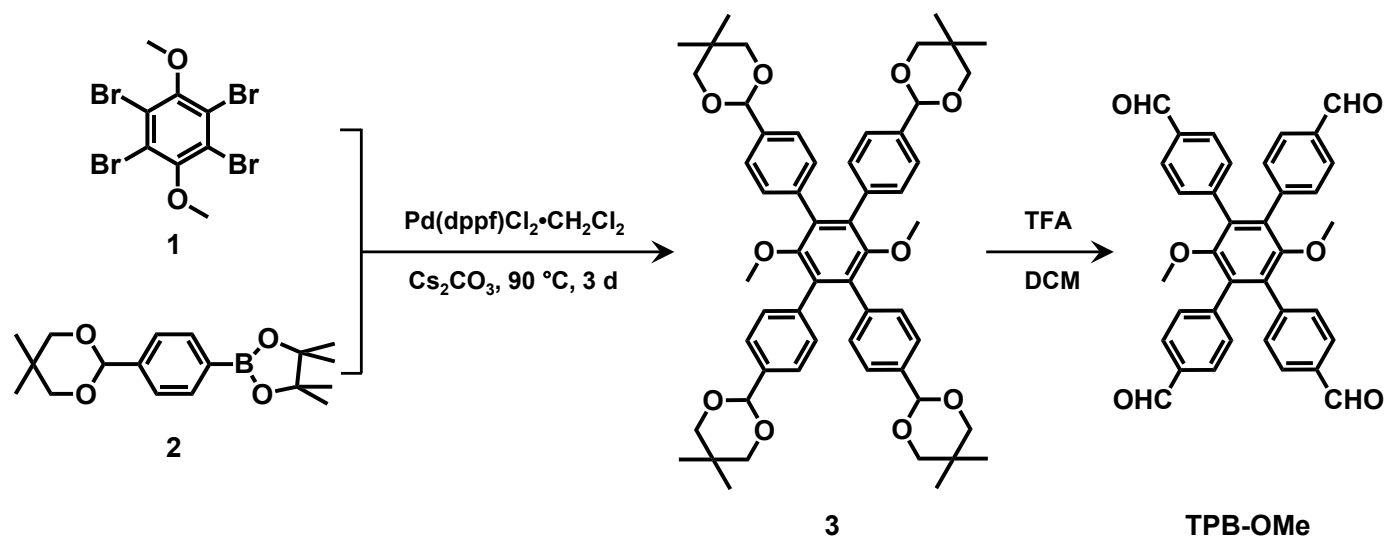

Scheme S1 Synthesis of TPB-OMe

Synthesis of Compound 3: A suspension of compound 1 (2.00 g, $4.4 \mathrm{mmol})$, compound 2 (7.00 g, $22 \mathrm{mmol}), \mathrm{Pd}(\mathrm{dppf}) \mathrm{Cl}_{2} \cdot \mathrm{CH}_{2} \mathrm{Cl}_{2}(0.36 \mathrm{~g}, 0.43 \mathrm{mmol})$ and cesium carbonate $(11.40 \mathrm{~g}, 35.1 \mathrm{mmol})$ in toluene $/ \mathrm{H}_{2} \mathrm{O}(100 \mathrm{~mL} / 10 \mathrm{~mL})$ was heated under nitrogen at $90{ }^{\circ} \mathrm{C}$ for 3 days. After cooling to room temperature, the solvents were removed under reduced pressure. The residues were dissolved in $\mathrm{CH}_{2} \mathrm{Cl}_{2}$, washed with water and brine, and dried over anhydrous $\mathrm{Na}_{2} \mathrm{SO}_{4}$. After that, the solvent was evaporated under reduced pressure and the crude product was purified by column chromatography $\left[\mathrm{SiO}_{2}: \mathrm{CH}_{2} \mathrm{Cl}_{2}\right.$ ] to yield compound $\mathbf{3}$ as a white solid (2.8 g, $70 \%$ yield). ${ }^{1} \mathrm{H} \mathrm{NMR}\left(400 \mathrm{MHz}, \mathrm{CDCl}_{3}, \mathrm{ppm}\right): \delta=7.31(\mathrm{~d}, J=8.0 \mathrm{~Hz}, 8 \mathrm{H}), 7.21(\mathrm{~d}, J=8.0 \mathrm{~Hz}$, 8H), $5.31(\mathrm{~s}, 4 \mathrm{H}), 3.75(\mathrm{~d}, J=12.0 \mathrm{~Hz}, 8 \mathrm{H}), 3.62(\mathrm{~d}, J=12.0 \mathrm{~Hz}, 8 \mathrm{H}), 2.73(\mathrm{~s}, 6 \mathrm{H})$, $1.28(\mathrm{~s}, 12 \mathrm{H}), 0.78(\mathrm{~s}, 12 \mathrm{H}) .{ }^{13} \mathrm{C} \mathrm{NMR}\left(100 \mathrm{MHz}, \mathrm{CDCl}_{3}, \mathrm{ppm}\right): \delta=151.9,137.2,136.6$, 135.5, 131.2, 125.5, 102.1, 77.8, 60.1, 30.4, 23.2, 22.1. HR-MS (MALDI-TOF): calcd for $\mathrm{C}_{56} \mathrm{H}_{66} \mathrm{O}_{10} \mathrm{~m} / \mathrm{z}=898.4656[\mathrm{M}]^{+}$, found: $\mathrm{m} / \mathrm{z}=898.4644[\mathrm{M}]^{+}$. 


\section{Synthesis of 1,2,4,5-tetrakis-(4-formylphenyl)-3,6-dimethoxybenzene (TPB-OMe).}

Trifluoroacetic acid $(5 \mathrm{~mL})$ was added dropwise into a solution of compound $\mathbf{3}$ (2.80 g, $3.1 \mathrm{mmol})$ in $\mathrm{CH}_{2} \mathrm{Cl}_{2}(20 \mathrm{~mL})$ and the mixture was stirred overnight at room temperature. Subsequently, the reaction was quenched by adding saturated sodium carbonate solution to $\mathrm{pH}=10$. The mixture was extracted with $\mathrm{CH}_{2} \mathrm{Cl}_{2}$ and washed with brine and dried over anhydrous $\mathrm{Na}_{2} \mathrm{SO}_{4}$. After that, the solvent was removed under vacuum to give TPB-OMe as a white solid (1.5 g, 86\% yield). ${ }^{1} \mathrm{H}$ NMR (400 MHz, $\left.\mathrm{CDCl}_{3}, \mathrm{ppm}\right): \delta=9.95(\mathrm{~s}, 4 \mathrm{H}), 7.74(\mathrm{~d}, J=8.0 \mathrm{~Hz}, 8 \mathrm{H}), 7.38(\mathrm{~d}, J=8.0 \mathrm{~Hz}, 8 \mathrm{H}), 2.87$ (s, 6H). ${ }^{13} \mathrm{C} \mathrm{NMR}\left(100 \mathrm{MHz}, \mathrm{CDCl}_{3}, \mathrm{ppm}\right): \delta=192.0,151.6,142.4,135.5,135.1,131.7$, 129.2, 60.5. HR-MS (MALDI-TOF): calcd for $\mathrm{C}_{36} \mathrm{H}_{26} \mathrm{O}_{6} \mathrm{~m} / \mathrm{z}=554.1729$ [M] $]^{+}$, found: $\mathrm{m} / \mathrm{z}=554.1720[\mathrm{M}]^{+}$.

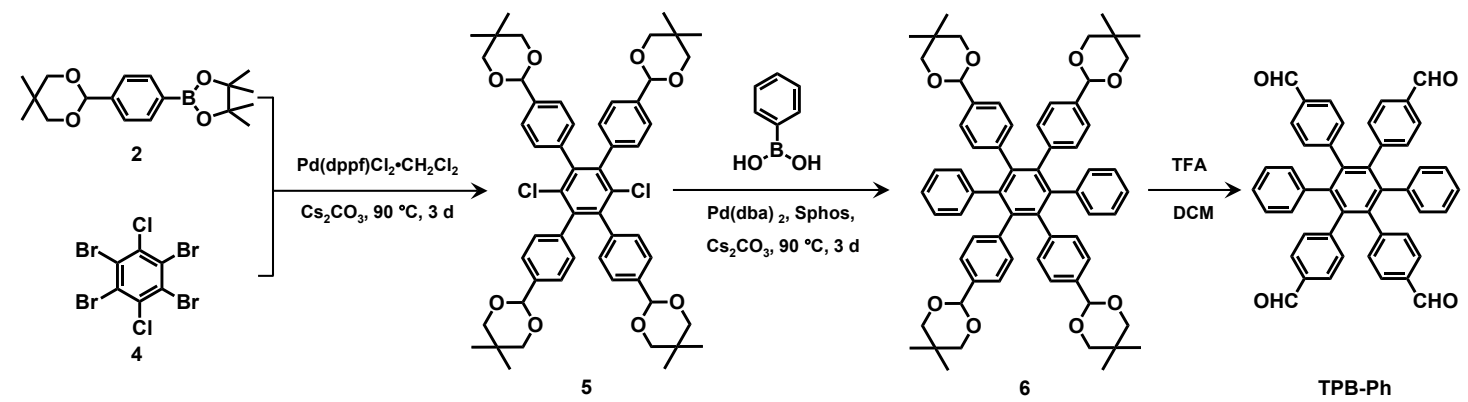

Scheme S2 Synthesis of TPB-Ph

Synthesis of Compound 5. A flask was charged with compound 2 (21.60 g, 67.9 mmol), compound 4 (6.00 g, $13.0 \mathrm{mmol}), \mathrm{Pd}(\mathrm{dppf}) \mathrm{Cl}_{2} \cdot \mathrm{CH}_{2} \mathrm{Cl}_{2}(1.08 \mathrm{~g}, 1.3 \mathrm{mmol})$, cesium carbonate $(34.80 \mathrm{~g}, 107.1 \mathrm{mmol})$ and toluene $/ \mathrm{H}_{2} \mathrm{O}(300 \mathrm{~mL} / 30 \mathrm{~mL})$. The mixture was heated under stirring at $90{ }^{\circ} \mathrm{C}$ for 3 days. After cooling down, the solvent was removed under reduced pressure. Then the residues were dissolved in $\mathrm{CH}_{2} \mathrm{Cl}_{2}$, washed with water and brine, and dried over anhydrous $\mathrm{Na}_{2} \mathrm{SO}_{4}$. After that, the solvent was evaporated under reduced pressure and the crude product was purified by column chromatography $\left[\mathrm{SiO}_{2}: \mathrm{CH}_{2} \mathrm{Cl}_{2} /\right.$ petroleum ether $=3 / 1$ ] to yield compound $\mathbf{5}$ as a white solid (8.3 g, 70\% yield). ${ }^{1} \mathrm{H}$ NMR (400 MHz, $\left.\mathrm{CDCl}_{3}, \mathrm{ppm}\right): \delta=7.32$ (d, $J=8.0 \mathrm{~Hz}$, $8 \mathrm{H}), 7.10(\mathrm{~d}, J=8.0 \mathrm{~Hz}, 8 \mathrm{H}), 5.29(\mathrm{~s}, 4 \mathrm{H}), 3.74(\mathrm{~d}, J=12.0 \mathrm{~Hz}, 8 \mathrm{H}), 3.60(\mathrm{~d}, J=12.0$ $\mathrm{Hz}, 8 \mathrm{H}), 1.26$ (s, 12H), 0.77(s, 12H). ${ }^{13} \mathrm{C} \mathrm{NMR}\left(100 \mathrm{MHz}, \mathrm{CDCl}_{3}, \mathrm{ppm}\right): \delta=141.1$, 
139.2, 137.2, 132.6, 130.2, 125.7, 101.8, 77.8, 30.4, 23.2, 22.0. HR-MS (MALDI-TOF): calcd for $\mathrm{C}_{54} \mathrm{H}_{60} \mathrm{Cl}_{2} \mathrm{NaO}_{8} \mathrm{~m} / \mathrm{z}=929.3563[\mathrm{M}+\mathrm{Na}]^{+}$, found: $\mathrm{m} / \mathrm{z}=929.3549[\mathrm{M}+\mathrm{Na}]^{+}$.

Synthesis of Compound 6. A suspension of compound 5 (3.00 g, $3.3 \mathrm{mmol})$, phenylboronic acid (6.00 g, $49.2 \mathrm{mmol}), \mathrm{Pd}(\mathrm{dba})_{2}(0.75 \mathrm{~g}, 1.3 \mathrm{mmol})$, Sphos (1.00 g, $2.4 \mathrm{mmol})$ and cesium carbonate $(3.00 \mathrm{~g}, 9.2 \mathrm{mmol})$ in toluene $(150 \mathrm{~mL})$ was heated under stirring at $90{ }^{\circ} \mathrm{C}$ for 3 days. After cooling to room temperature, the solvent was removed under reduced pressure. Then the residues were dissolved in $\mathrm{CH}_{2} \mathrm{Cl}_{2}$, washed with water and brine, and dried over anhydrous $\mathrm{Na}_{2} \mathrm{SO}_{4}$. After that, the solvent was evaporated under reduced pressure and the crude product was purified by column chromatography $\left[\mathrm{SiO}_{2}: \mathrm{CH}_{2} \mathrm{Cl}_{2} /\right.$ petroleum ether $=3 / 1$ ] to yield compound $\mathbf{6}$ as a white solid (2.1 g, 65\% yield). ${ }^{1} \mathrm{H}$ NMR (400 MHz, $\left.\mathrm{CDCl}_{3}, \mathrm{ppm}\right): \delta=6.97(\mathrm{~d}, J=8.0 \mathrm{~Hz}$, $8 \mathrm{H}), 6.85-6.78(\mathrm{~m}, 14 \mathrm{H}), 6.77-6.72(\mathrm{~m}, 4 \mathrm{H}), 5.13(\mathrm{~s}, 4 \mathrm{H}), 3.65(\mathrm{~d}, J=12.0 \mathrm{~Hz}, 8 \mathrm{H})$, $3.50(\mathrm{~d}, J=12.0 \mathrm{~Hz}, 8 \mathrm{H}), 1.19(\mathrm{~s}, 12 \mathrm{H}), 0.73(\mathrm{~s}, 12 \mathrm{H}) .{ }^{13} \mathrm{C} \mathrm{NMR}\left(100 \mathrm{MHz}, \mathrm{CDCl}_{3}\right.$, ppm): $\delta=141.2,140.8,140.4,140.2,135.5,131.5,126.9,125.5,124.7,101.9,77.6$, 30.3, 23.1, 22.0. HR-MS (MALDI-TOF): calcd for $\mathrm{C}_{66} \mathrm{H}_{71} \mathrm{O}_{8} \mathrm{~m} / \mathrm{z}=991.5149[\mathrm{M}+\mathrm{H}]^{+}$, found: $\mathrm{m} / \mathrm{z}=991.5138[\mathrm{M}+\mathrm{H}]^{+}$.

\section{Synthesis of 1,2,4,5-tetrakis-(4-formylphenyl)-3,6-diphenylbenzene (TPB-Ph).} Trifluoroacetic acid $(5 \mathrm{~mL})$ was added into a solution of compound $\mathbf{6}(1.20 \mathrm{~g}, 1.2 \mathrm{mmol})$ in $\mathrm{CH}_{2} \mathrm{Cl}_{2}(20 \mathrm{~mL})$, and then the mixture was stirred overnight at room temperature. Subsequently, the reaction was quenched by adding saturated sodium carbonate solution to $\mathrm{pH}=10$. The mixture was extracted with $\mathrm{CH}_{2} \mathrm{Cl}_{2}$ and washed with brine and dried over anhydrous $\mathrm{Na}_{2} \mathrm{SO}_{4}$. After that, the solvent was removed under vacuum to give TPB-Ph as a white solid (0.74 g, $95 \%$ yield). ${ }^{1} \mathrm{H}$ NMR (400 MHz, $\mathrm{CDCl}_{3}$, ppm): $\delta=9.76(\mathrm{~s}, 4 \mathrm{H}), 7.41(\mathrm{~d}, J=8.0 \mathrm{~Hz}, 8 \mathrm{H}), 7.01(\mathrm{~d}, J=8.0 \mathrm{~Hz}, 8 \mathrm{H}), 6.90-6.85(\mathrm{~m}, 6 \mathrm{H})$, 6.85-6.75 (m, 4H). ${ }^{13} \mathrm{C}$ NMR (100 MHz, $\left.\mathrm{CDCl}_{3}, \mathrm{ppm}\right): \delta=192.0,146.4,140.4,139.8$, 138.7, 133.9, 131.8, 131.0, 128.6, 127.5, 126.6. HR-MS (MALDI-TOF): calcd for $\mathrm{C}_{46} \mathrm{H}_{30} \mathrm{NaO}_{4} \mathrm{~m} / \mathrm{z}=669.2036[\mathrm{M}+\mathrm{Na}]^{+}$, found: $\mathrm{m} / \mathrm{z}=669.2037[\mathrm{M}+\mathrm{Na}]^{+}$. 


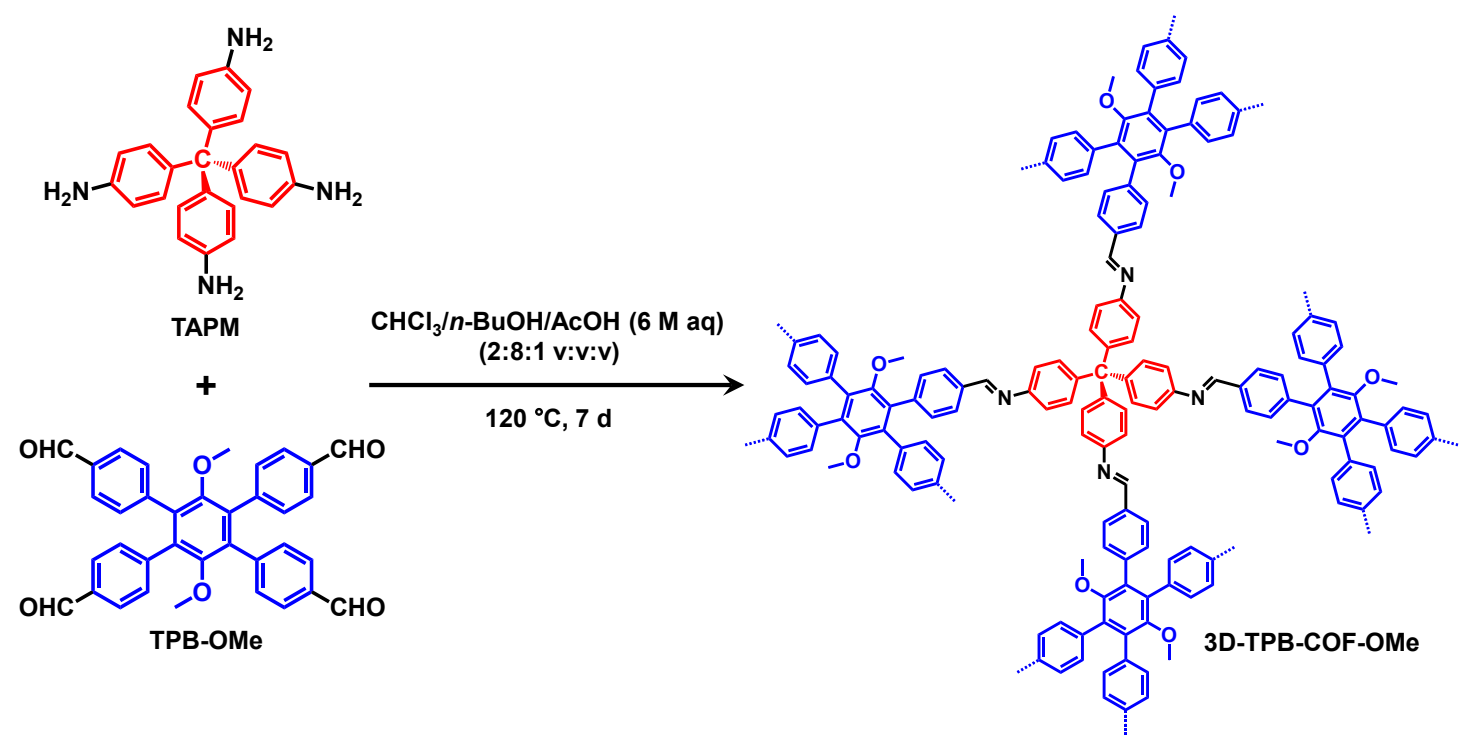

Scheme S3 Synthesis of 3D-TPB-COF-OMe

Synthesis of 3D-TPB-COF-OMe. A Pyrex tube was charged with TAPM (15.2 mg, $0.04 \mathrm{mmol})$, TPB-OMe (21.6 mg, $0.04 \mathrm{mmol})$, chloroform (0.4 mL), $n$-BuOH (1.6 mL) and $6 \mathrm{M}$ aqueous acetic acid $(0.2 \mathrm{~mL})$. After being degassed by freeze-pump-thaw technique for three times and then sealed under vacuum, the tube was placed in an oven at $120{ }^{\circ} \mathrm{C}$ for $7 \mathrm{~d}$. The resulting precipitate was filtered, washed with DMF, tetrahydrofuran and dichloromethane for $2 \mathrm{~d}$, dried at $120{ }^{\circ} \mathrm{C}$ under vacuum for $12 \mathrm{~h}$. The activated 3D-TPB-COF-OMe was obtained as a white powder $(20.3 \mathrm{mg}, 59 \%$ yield). Elemental analysis for the calculated: C, 84.89\%; H, 4.91\%; N, 6.49\%. Found: C, 81.34\%; H, 5.84\%; N, 5.48\%. 


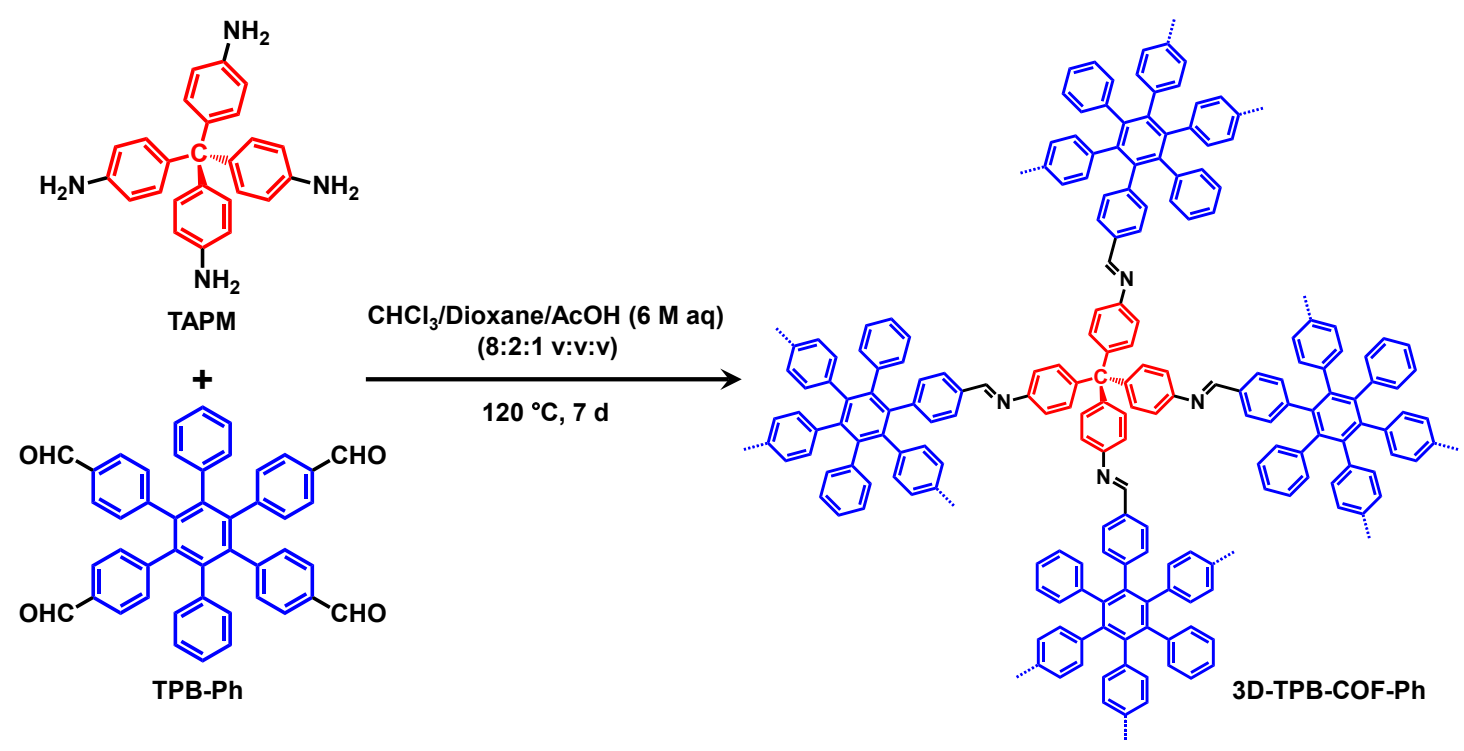

Scheme S4 Synthesis of 3D-TPB-COF-Ph.

Synthesis of 3D-TPB-COF-Ph. A Pyrex tube was charged with TAPM (15.2 mg, 0.04 mmol), TPB-Ph (26.0 mg, $0.04 \mathrm{mmol})$, chloroform $(3.2 \mathrm{~mL})$, dioxane $(0.8 \mathrm{~mL})$ and 6 $\mathrm{M}$ aqueous acetic acid $(0.4 \mathrm{~mL})$. After being degassed by freeze-pump-thaw technique for three times and then sealed under vacuum, the tube was placed in an oven at $120{ }^{\circ} \mathrm{C}$ for $7 \mathrm{~d}$. The resulting precipitate was filtered, washed with DMF, tetrahydrofuran and dichloromethane for $2 \mathrm{~d}$, dried at $100{ }^{\circ} \mathrm{C}$ under vacuum for $12 \mathrm{~h}$. The activated 3DTPB-COF-Ph was obtained as a white powder (35.6 mg, 93\% yield). Elemental analysis for the calculated: $\mathrm{C}, 89.28 \% ; \mathrm{H}, 4.85 \% ; \mathrm{N}, 5.87 \%$. Found: C, 87.56\%; H, 4.79\%; N, $5.79 \%$.

Optimizing the reaction condition for 3D-TPB-COF-Ph: In order to synthesize 3DTPB-COF-Ph with high crystallinity toward cRED technique, we have tried many different reaction conditions by changing solvents, the concentration of acid and reactants, etc.. We should mention here, the obtained crystalline samples in these conditions don't exhibit significant difference in PXRD patterns, except the peak intensity. As shown in Figure S1, by using the same synthetic condition for 3D-TPBCOF-OMe, we can also synthesize 3D-TPB-COF-Ph but with low crystallinity. Therefore, the possibility of solvent effect on the formation of 3D-TPB-COF-OMe and 
3D-TPB-COF-Ph can be excluded. Finally, after many trials, we get the right condition to synthesize high crystalline 3D-TPB-COF-Ph toward cRED technique.

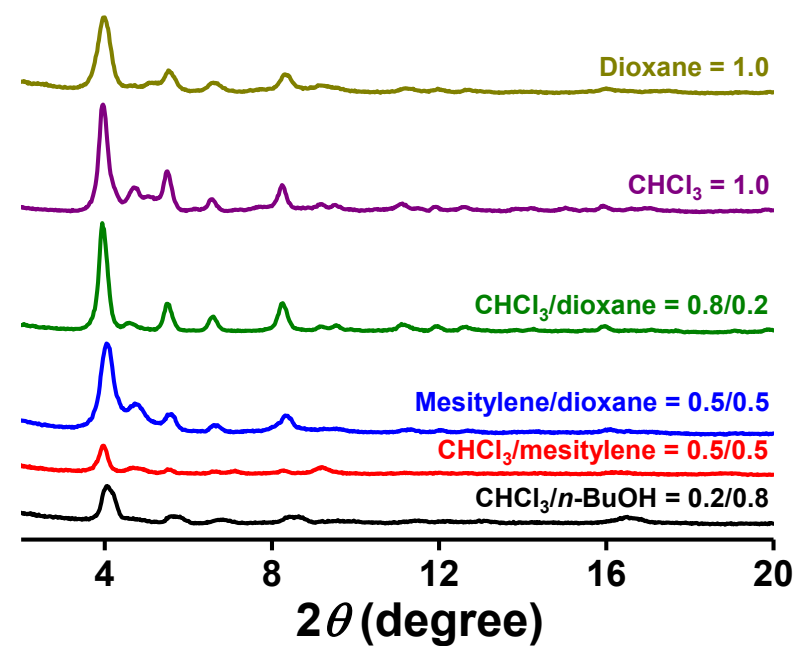

Figure S1 PXRD patterns of 3D-TPB-COF-Ph obtained in different solvent conditions (6 M acetic acid: $0.1 \mathrm{~mL}$; Temp: $120^{\circ} \mathrm{C}$ ). Data was collected on Rigaku MiniFlex 600 X-Ray diffractometer.

\section{Section S3. Characterizations of 3D COFs}

\section{A. FT-IR Spectroscopy Analysis}

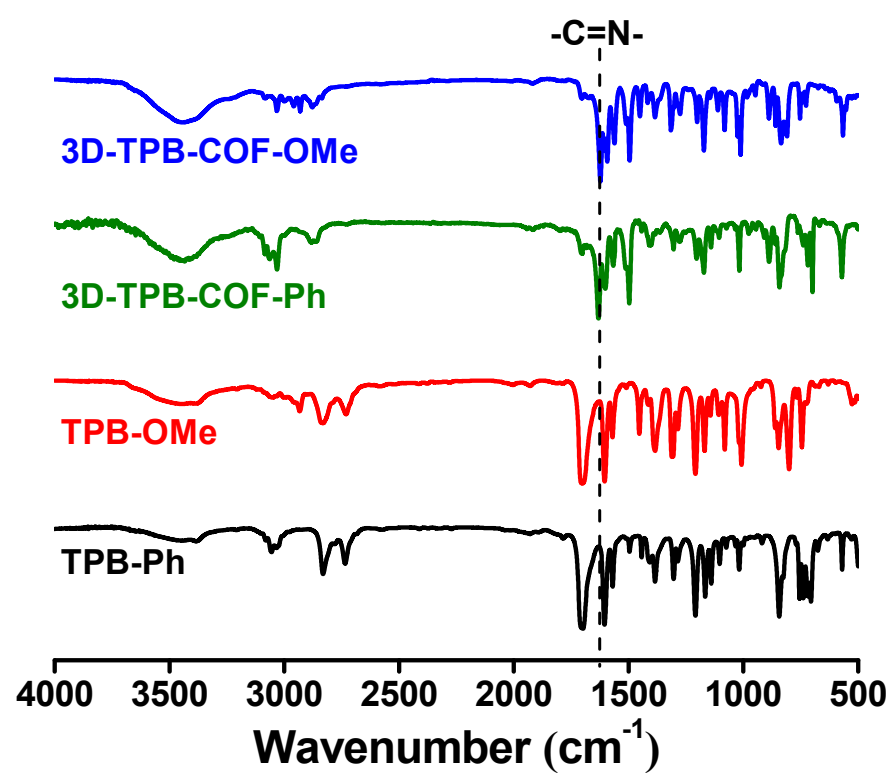

Figure S2 FT-IR spectra of TPB-Ph (black curve), TPB-OMe (red curve), 3D-TPBCOF-Ph (green curve) and 3D-TPB-COF-OMe (blue curve). 


\section{B. ${ }^{13}$ C Solid-State NMR Spectroscopy}



Figure $\mathbf{S 3}{ }^{13} \mathrm{C}$ solid-state NMR spectra of 3D-TPB-COF-OMe

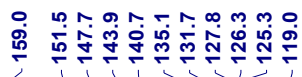

ஸे



Figure S4 ${ }^{13} \mathrm{C}$ solid-state NMR spectra of 3D-TPB-COF-Ph

\section{Scanning electron microscopy}
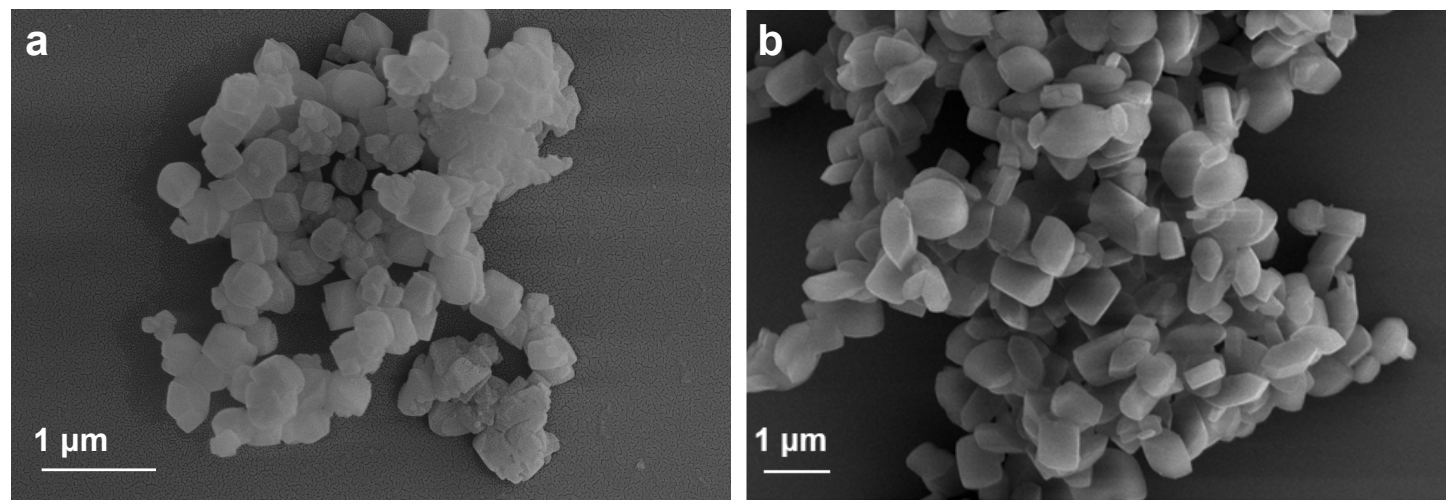

Figure S5 SEM images of the 3D-TPB-COF-OMe (a) and the 3D-TPB-COF-Ph (b). 
D. $\mathbf{N}_{2}$ sorption isotherms

(a)

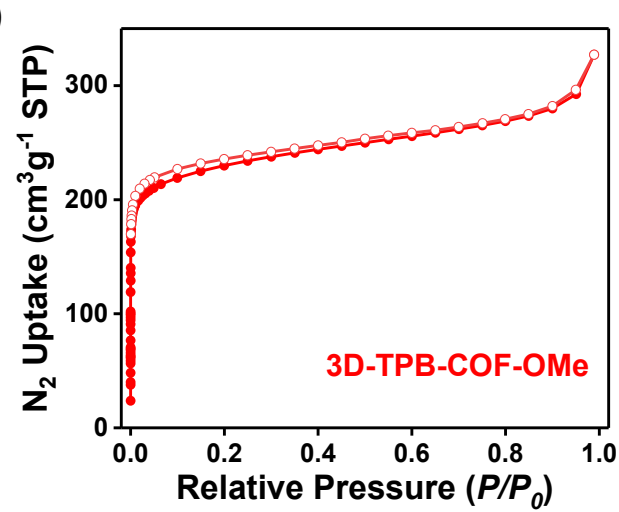

(c)

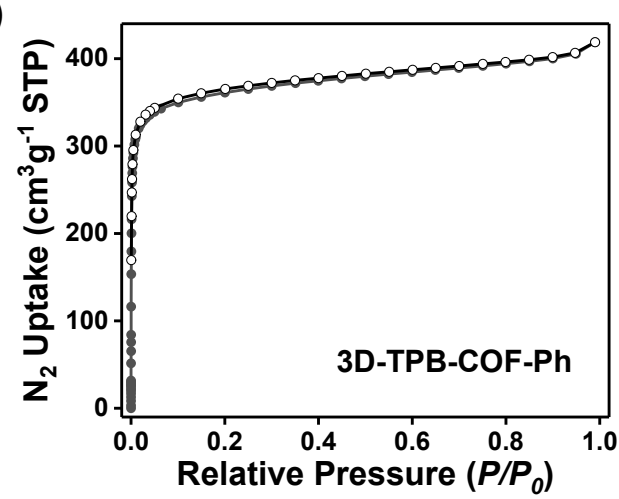

(b)

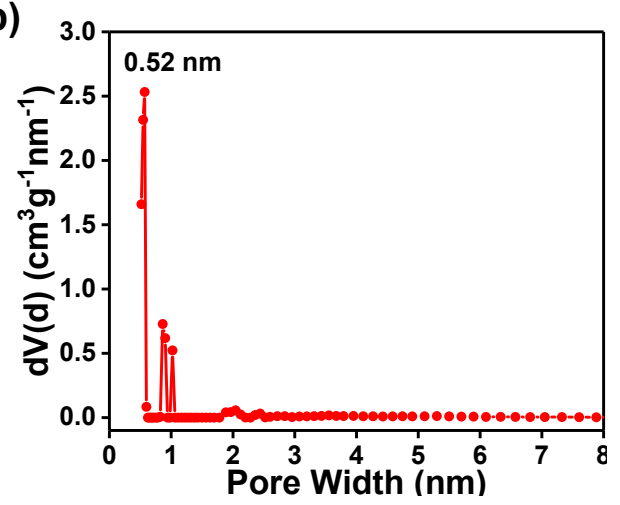

(d)

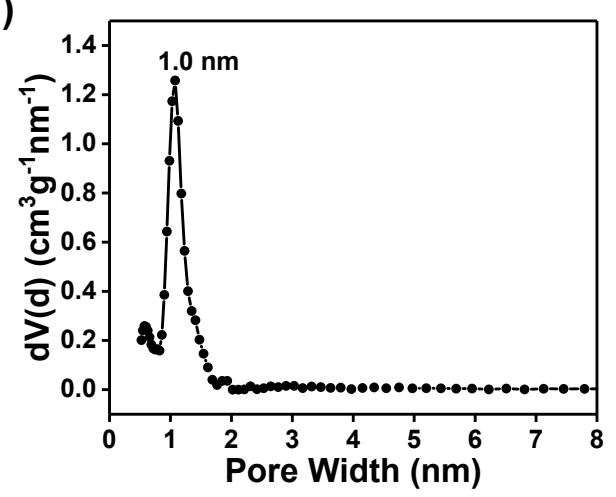

Figure $\mathrm{S6}_{2}$ sorption isotherm of 3D-TPB-COF-OMe (a) and 3D-TPB-COF-Ph (c) at 77K. Pore size distribution of 3D-TPB-COF-OMe (b) and 3D-TPB-COF-Ph (d)

E. Thermogravimetric Analysis

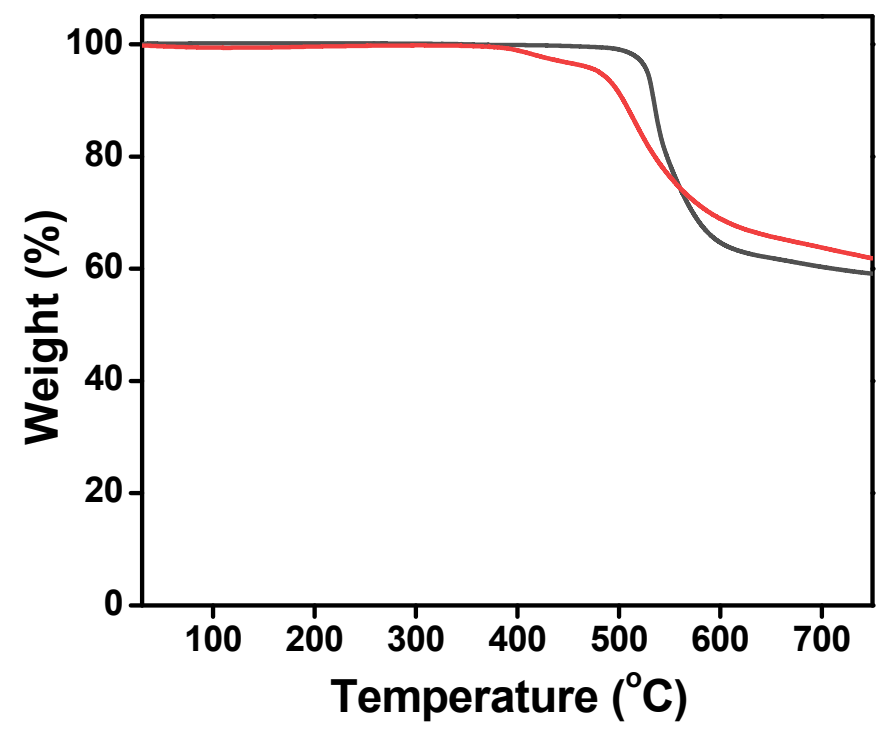

Figure S7 TGA profile of 3D-TPB-COF-OMe (red curve) and 3D-TPB-COF-Ph (black curve) in nitrogen atmosphere. 


\section{F. Chemical Stability}

In a typical experiment, $10 \mathrm{mg}$ of 3D-TPB-COF-OMe or 3D-TPB-COF-Ph was immersed in different solvents (DMF, DMSO, acetone, ethanol, acetonitrile, toluene, $n$-hexane, 1,4-dioxane, ethyl acetate, $0.1 \mathrm{M} \mathrm{HCl}, 12 \mathrm{M} \mathrm{NaOH}$ ) for $24 \mathrm{~h}$ at room temperature. After that, the powder was filtrated, washed with tetrahydrofuran and dichloromethane, then dried under vacuum to measure PXRD patterns. Data collected on Rigaku MiniFlex 600 X-Ray diffractometer.

(a)

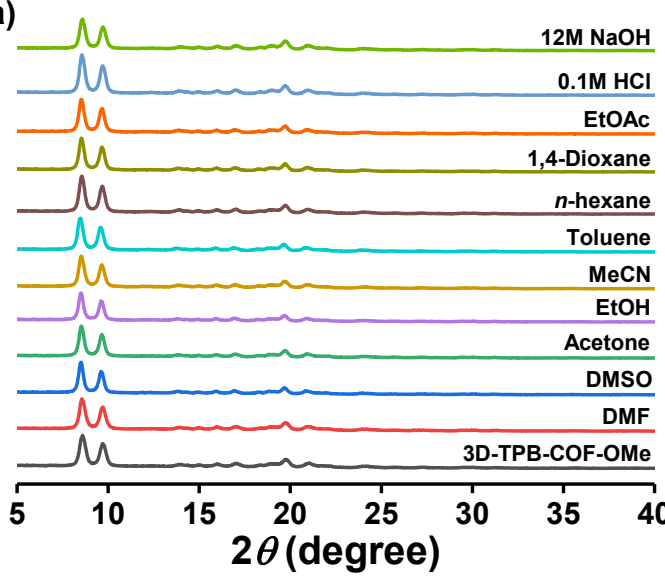

(b)

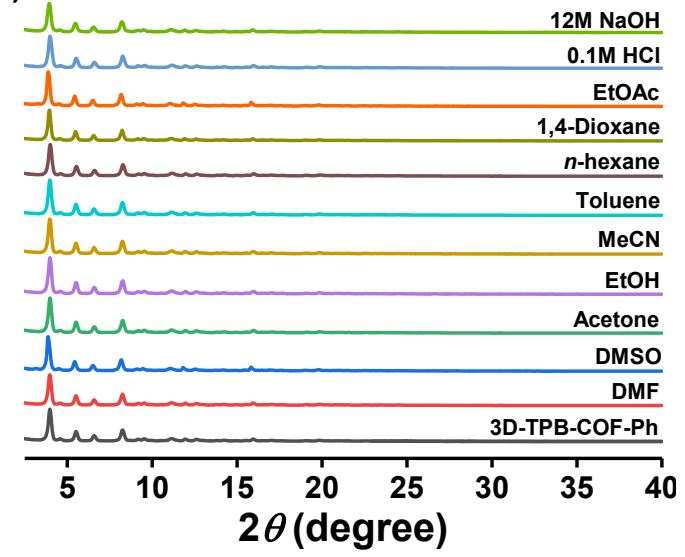

Figure S8 The PXRD patterns of 3D-TPB-COF-OMe (a) and 3D-TPB-COF-Ph (b) before and after being treated in different solvents for $24 \mathrm{~h}$.

\section{G. Variable temperature PXRD experiments}

(a)

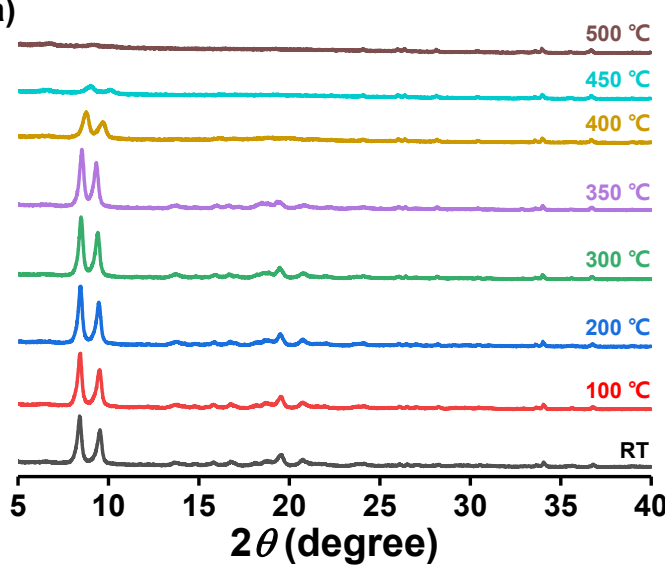

(b)

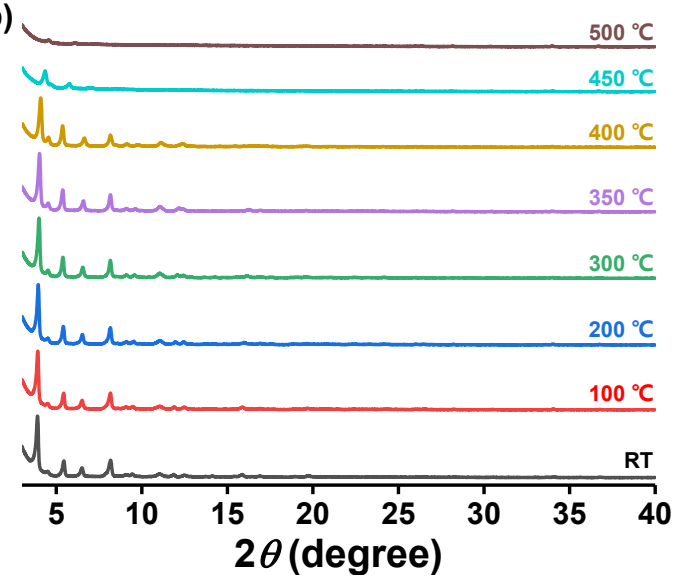

Figure S9 In situ PXRD patterns of 3D-TPB-COF-OMe (a) and 3D-TPB-COF-Ph (b) at variable temperature. 


\section{Section S4. Crystal structure analysis}

The method of cRED data collection was similar with our previous report ${ }^{\mathrm{S} 5}$. Shortly, the sample was cooled down to $99 \mathrm{~K}$ by using Gatan cryo-transfer tomography holder and the data was collected by a quad hybrid pixel detector (Timepix) with video mode, using the software of instamatic ${ }^{\mathrm{S} 6}$. During the data collection, the goniometer was rotated continuously while the selected area ED patterns were captured from the crystal simultaneous, and all the ED patterns were recorded under the spot size 3 with the exposure time $0.5 \mathrm{~s}$. Data processing was conducted using the software package XDS ${ }^{\mathrm{S} 7}$ and REDp ${ }^{\mathrm{S} 8}$. Structure solution were performed by SHELXT ${ }^{\mathrm{S} 9}$ with the merged and scaled datasets. With the hkl file, we can perform the structure solution by direct methods, charge flipping, etc., the same as single crystal X-ray diffraction. PXRD patterns were obtained on a Rigaku SmartLab X-Ray diffractometer with $\mathrm{Cu} \mathrm{K} \alpha$ line $(\lambda$ $=1.5418 \AA$ ) focused radiation at $45 \mathrm{kV}$ and $200 \mathrm{~mA}$. The Rietveld refinement with rigid-body constraints was performed on the experimental PXRD using Topas $4.1^{\text {S10. }}$

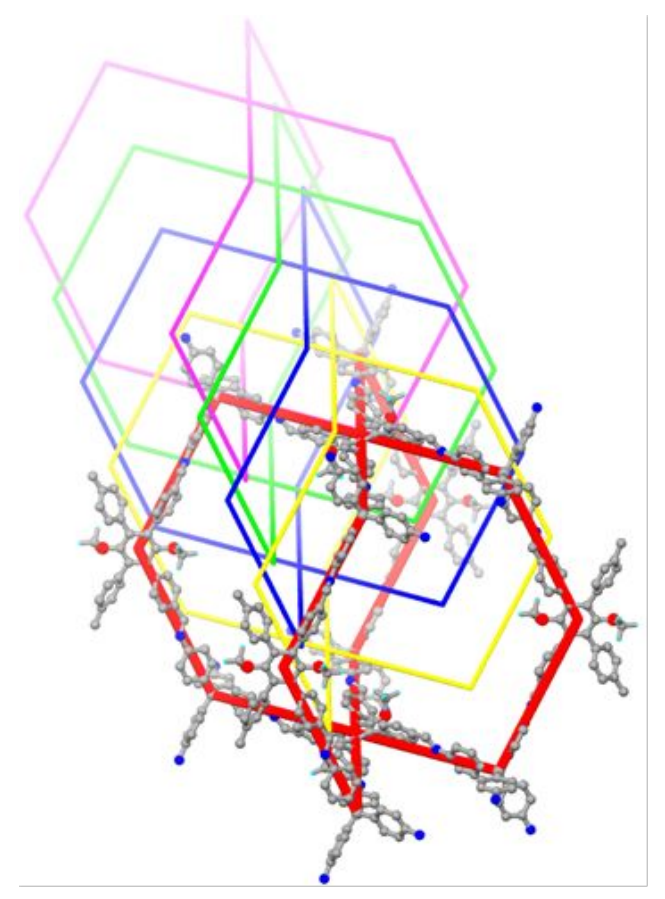

Figure S10 The 5-fold interpenetrated pts net of 3D-TPB-COF-OMe. The carbon is colored in dim gray, nitrogen is in blue, oxygen is in red and hydrogen is in cyan; all $\mathrm{H}$ atoms are omitted for clarity except for the $\mathrm{H}$ atoms in the methoxy groups. 


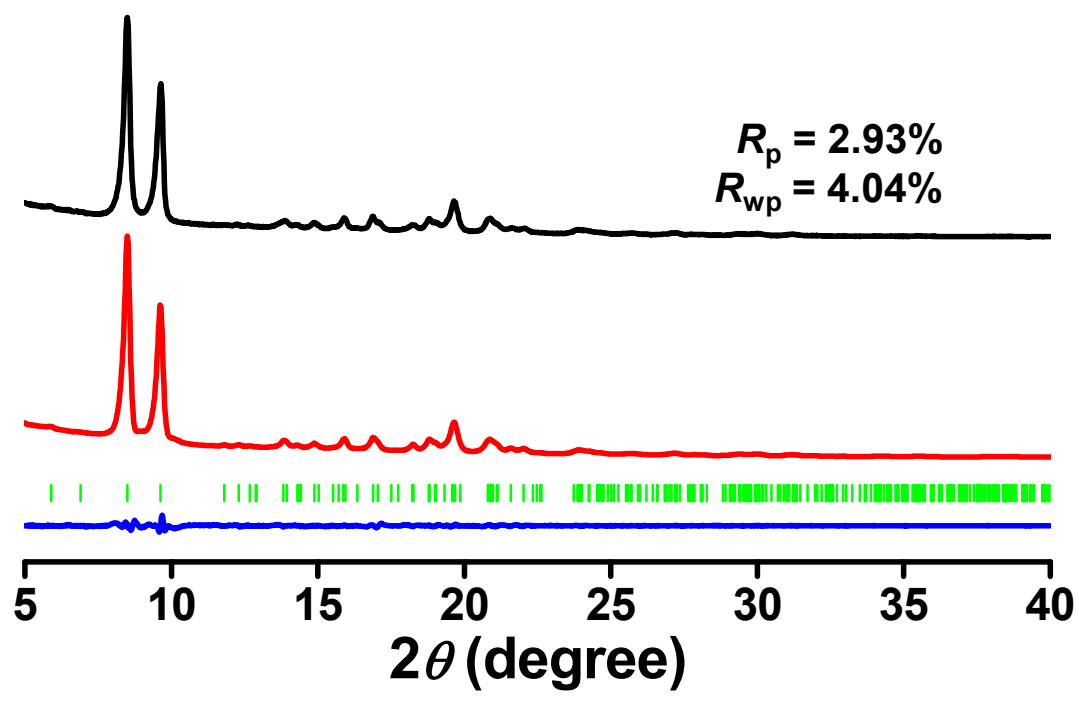

Figure S11 Powder X-ray diffraction patterns of 3D-TPB-COF-OMe. The experimental PXRD patterns are shown in black, the refined patterns in red, bragg position from Rietveld refinement patterns in green and the difference between the experimental and refined profiles in blue.

Table S1 Summary of the Rietveld refinement results of 3D-TPB-COF-OMe.

\begin{tabular}{|c|c|}
\hline Name & 3D-TPB-COF-OMe \\
\hline Chemical formula & $\mathrm{C}_{31} \mathrm{H}_{21} \mathrm{~N}_{2} \mathrm{O}$ \\
\hline Formula Weight/ $\mathrm{g} \mathrm{mol}^{-1}$ & 437.51 \\
\hline Density $/ \mathrm{g} \mathrm{cm}^{-3}$ & $1.007(3)$ \\
\hline Crystal system & Monoclinic \\
\hline Unit cell & $\begin{array}{c}a=30.296(1) \AA, b=7.431(3) \AA, c= \\
25.464(6) \AA \\
\alpha=\gamma=90^{\circ}, \beta=96.974(2)^{\circ}\end{array}$ \\
\hline Space group & $C 2 / c$ \\
\hline Volume $/ \AA^{3}$ & 5690.65 \\
\hline $\mathrm{Z}$ & 8 \\
\hline X-ray source & $\mathrm{Cu} K \alpha$ \\
\hline Wavelength /Å & 1.5418 \\
\hline $2 \theta$ & $2-40^{\circ}$ \\
\hline Number of reflections & 285 \\
\hline
\end{tabular}




\begin{tabular}{|c|c|}
\hline Number of data points & 1901 \\
\hline Refinement method & Rietveld refinement \\
\hline$R_{p}$ & $2.93 \%$ \\
\hline$R_{w p}$ & $4.04 \%$ \\
\hline GoF & 1.97 \\
\hline
\end{tabular}

Table S2 Fractional atomic coordinates and the unit cell of 3D-TPB-COF-OMe from the Rietveld refinement.

\begin{tabular}{|c|c|c|c|c|}
\hline \multicolumn{2}{|c|}{ 3D-TPB-COF-OMe } & \multicolumn{3}{|c|}{$\begin{array}{c}\text { Space-group: } C 2 / c \\
a=30.296(1) \AA, b=7.431(3) \AA, c=25.464(6) \AA \\
\alpha=\gamma=90^{\circ}, \beta=96.974(2)^{\circ}\end{array}$} \\
\hline $\mathrm{C} 1$ & $\mathrm{C}$ & 0.53261 & 0.51983 & 0.28335 \\
\hline $\mathrm{C} 2$ & $\mathrm{C}$ & -0.02445 & 0.76466 & 0.28513 \\
\hline $\mathrm{C} 3$ & $\mathrm{C}$ & -0.07126 & 0.7572 & 0.28234 \\
\hline $\mathrm{C} 4$ & $\mathrm{C}$ & -0.06726 & 0.50347 & 0.34209 \\
\hline $\mathrm{C} 5$ & $\mathrm{C}$ & 0.55761 & 0.64219 & 0.25658 \\
\hline C6 & $\mathrm{C}$ & 0.00033 & 0.63726 & 0.31777 \\
\hline $\mathrm{C} 7$ & $\mathrm{C}$ & -0.09203 & 0.63056 & 0.31104 \\
\hline N8 & $\mathrm{N}$ & 0.61571 & 0.91176 & 0.36869 \\
\hline C9 & $\mathrm{C}$ & 0.5361 & 0.53198 & 0.33914 \\
\hline $\mathrm{C} 10$ & $\mathrm{C}$ & 0.58927 & 0.77656 & 0.33939 \\
\hline $\mathrm{C} 11$ & $\mathrm{C}$ & 0.56458 & 0.65723 & 0.36661 \\
\hline N12 & $\mathrm{N}$ & -0.09146 & 0.36497 & 0.36499 \\
\hline $\mathrm{C} 13$ & $\mathrm{C}$ & -0.02071 & 0.50873 & 0.34617 \\
\hline $\mathrm{C} 14$ & $\mathrm{C}$ & 0.58648 & 0.76422 & 0.28409 \\
\hline $\mathrm{C} 15$ & $\mathrm{C}$ & 0.2249 & 0.88491 & 0.97101 \\
\hline $\mathrm{C} 16$ & $\mathrm{C}$ & 0.26106 & 0.80388 & 0.95035 \\
\hline $\mathrm{C} 17$ & $\mathrm{C}$ & 0.28621 & 0.66958 & 0.97903 \\
\hline $\mathrm{C} 18$ & $\mathrm{C}$ & 0.31947 & 0.50538 & 1.11159 \\
\hline C19 & $\mathrm{C}$ & 0.13325 & 0.9157 & 1.01812 \\
\hline $\mathrm{C} 20$ & $\mathrm{C}$ & 0.17672 & 0.91528 & 1.04466 \\
\hline $\mathrm{C} 21$ & $\mathrm{C}$ & 0.1855 & 1.00209 & 1.09358 \\
\hline
\end{tabular}




\begin{tabular}{|c|c|c|c|c|}
\hline $\mathrm{C} 22$ & $\mathrm{C}$ & 0.15201 & 1.09614 & 1.11438 \\
\hline $\mathrm{C} 23$ & $\mathrm{C}$ & 0.10937 & 1.10945 & 1.08592 \\
\hline $\mathrm{C} 24$ & $\mathrm{C}$ & 0.09969 & 1.0133 & 1.03855 \\
\hline $\mathrm{C} 25$ & $\mathrm{C}$ & 0.34243 & 0.3702 & 1.14123 \\
\hline $\mathrm{C} 26$ & $\mathrm{C}$ & 0.34688 & 0.19883 & 1.11945 \\
\hline $\mathrm{C} 27$ & $\mathrm{C}$ & 0.3274 & 0.16335 & 1.06762 \\
\hline $\mathrm{C} 28$ & $\mathrm{C}$ & 0.30401 & 0.29867 & 1.03813 \\
\hline $\mathrm{C} 29$ & $\mathrm{C}$ & 0.30009 & 0.47091 & 1.05982 \\
\hline $\mathrm{C} 30$ & $\mathrm{C}$ & 0.07673 & 1.23467 & 1.10385 \\
\hline $\mathrm{C} 31$ & $\mathrm{C}$ & 0.3723 & 0.06165 & 1.15167 \\
\hline $\mathrm{O} 32$ & $\mathrm{O}$ & 0.27161 & 3.14584 & 0.40026 \\
\hline H33 & $\mathrm{H}$ & -0.0928 & 0.84264 & 0.25659 \\
\hline H34 & $\mathrm{H}$ & 0.55534 & 0.63987 & 0.21383 \\
\hline H35 & $\mathrm{H}$ & 0.0362 & 0.63611 & 0.32046 \\
\hline H36 & $\mathrm{H}$ & -0.12803 & 0.62603 & 0.30709 \\
\hline H37 & $\mathrm{H}$ & 0.51608 & 0.45024 & 0.3621 \\
\hline H38 & $\mathrm{H}$ & 0.56597 & 0.66623 & 0.40919 \\
\hline H39 & $\mathrm{H}$ & -0.00057 & 0.41263 & 0.37003 \\
\hline $\mathrm{H} 40$ & $\mathrm{H}$ & 0.60662 & 0.84751 & 0.26174 \\
\hline H41 & $\mathrm{H}$ & 0.31686 & 0.63733 & 1.129 \\
\hline H42 & $\mathrm{H}$ & 0.12589 & 0.84564 & 0.98084 \\
\hline H43 & $\mathrm{H}$ & 0.21875 & 1.00254 & 1.11477 \\
\hline H44 & $\mathrm{H}$ & 0.16004 & 1.1648 & 1.15183 \\
\hline H45 & $\mathrm{H}$ & 0.06688 & 1.01992 & 1.01638 \\
\hline H46 & $\mathrm{H}$ & 0.3571 & 0.40048 & 1.18109 \\
\hline H47 & $\mathrm{H}$ & 0.33009 & 0.03168 & 1.05001 \\
\hline $\mathrm{H} 48$ & $\mathrm{H}$ & 0.28907 & 0.2695 & 0.99838 \\
\hline H49 & $\mathrm{H}$ & 0.04185 & 1.22202 & 1.08914 \\
\hline H50 & $\mathrm{H}$ & 0.38333 & 0.0947 & 1.19241 \\
\hline C51 & $\mathrm{C}$ & 0.30332 & 3.00396 & 0.40183 \\
\hline H52 & $\mathrm{H}$ & 0.31087 & 2.97754 & 0.36101 \\
\hline H53 & $\mathrm{H}$ & 0.28948 & 2.87934 & 0.41729 \\
\hline
\end{tabular}




\begin{tabular}{|l|l|l|l|l|}
\hline H54 & H & 0.33476 & 3.04137 & 0.42659 \\
\hline C55 & $\mathrm{C}$ & 0.50000 & 0.3919 & 0.25000 \\
\hline
\end{tabular}

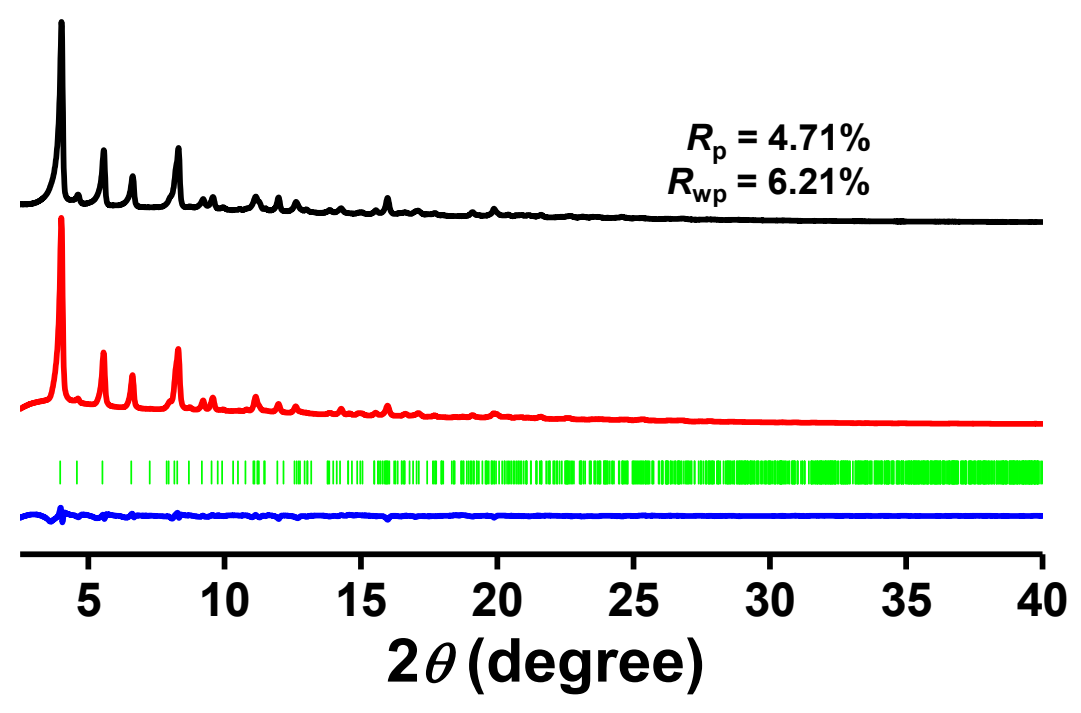

Figure S12 Powder X-ray diffraction patterns of 3D-TPB-COF-Ph. The experimental PXRD patterns are shown in black, the refined patterns in red, bragg position from Rietveld refinement patterns in green and the difference between the experimental and refined profiles in blue.

Table S3 Summary of the Rietveld refinement results of 3D-TPB-COF-Ph.

\begin{tabular}{|c|c|}
\hline Name & 3D-TPB-COF-Ph \\
\hline Chemical formula & $\mathrm{C}_{142} \mathrm{H}_{92} \mathrm{~N}_{8}$ \\
\hline Formula Weight/ $\mathrm{g} \mathrm{mol}^{-1}$ & 1910.30 \\
\hline Density $/ \mathrm{g} \mathrm{cm}^{-3}$ & $0.7241(7)$ \\
\hline Crystal system & Monoclinic \\
\hline Unit cell & $\begin{array}{c}\mathrm{a}=23.519(8) \AA, \mathrm{b}=44.466(6) \AA, \mathrm{c}= \\
18.527(4) \AA, \\
\alpha=\gamma=90^{\circ} \text {, and } \beta=114.564(8)^{\circ}\end{array}$ \\
\hline Space group & $C c$ \\
\hline Volume $/ \AA^{3}$ & 17521.8 \\
\hline $\mathrm{Z}$ & 4 \\
\hline X-ray source & $\mathrm{Cu} K \alpha$ \\
\hline
\end{tabular}




\begin{tabular}{|c|c|}
\hline Wavelength $/ \AA$ & 1.5418 \\
\hline $2 \theta$ & $2.5-40^{\circ}$ \\
\hline Number of reflections & 812 \\
\hline Number of data points & 3751 \\
\hline Refinement method & Rietveld refinement \\
\hline$R_{p}$ & $4.71 \%$ \\
\hline$R_{w p}$ & $6.21 \%$ \\
\hline GoF & 1.91 \\
\hline
\end{tabular}

Table S4 Fractional atomic coordinates and the unit cell of 3D-TPB-COF-Ph from the Rietveld refinement.

\begin{tabular}{|c|c|c|c|c|}
\hline \multicolumn{2}{|c|}{ 3D-TPB-COF-Ph } & \multicolumn{3}{|c|}{$\begin{array}{c}\text { Space-group: } C c \\
a=23.519(8) \AA, b=44.466(6) \AA, c=18.527(4) \AA, \\
\alpha=\gamma=90^{\circ} \text {, and } \beta=114.564(8)^{\circ}\end{array}$} \\
\hline $\mathrm{C} 1$ & $\mathrm{C}$ & -0.11432 & 0.54974 & 0.84941 \\
\hline $\mathrm{C} 2$ & $\mathrm{C}$ & 0.78926 & 0.57343 & 0.84333 \\
\hline $\mathrm{C} 3$ & $\mathrm{C}$ & 0.42426 & 0.56742 & 0.74778 \\
\hline $\mathrm{C} 4$ & $\mathrm{C}$ & 0.49039 & 0.56592 & 0.77696 \\
\hline $\mathrm{C} 5$ & $\mathrm{C}$ & 0.51899 & 0.5678 & 0.72328 \\
\hline C6 & $\mathrm{C}$ & 0.48139 & 0.56769 & 0.6401 \\
\hline $\mathrm{C} 7$ & $\mathrm{C}$ & 0.41532 & 0.56585 & 0.61094 \\
\hline $\mathrm{C} 8$ & $\mathrm{C}$ & 0.38663 & 0.56731 & 0.66458 \\
\hline $\mathrm{C} 9$ & $\mathrm{C}$ & -0.0276 & 0.5184 & 0.85663 \\
\hline $\mathrm{C} 10$ & $\mathrm{C}$ & 0.21653 & 0.5914 & 0.55364 \\
\hline $\mathrm{C} 11$ & $\mathrm{C}$ & 0.18517 & 0.56848 & 0.57458 \\
\hline $\mathrm{C} 12$ & $\mathrm{C}$ & 0.22043 & 0.54539 & 0.6257 \\
\hline $\mathrm{C} 13$ & $\mathrm{C}$ & 0.28556 & 0.54484 & 0.65396 \\
\hline C14 & $\mathrm{C}$ & 0.31697 & 0.56781 & 0.63344 \\
\hline $\mathrm{C} 15$ & $\mathrm{C}$ & 0.28198 & 0.59115 & 0.58311 \\
\hline C16 & $\mathrm{C}$ & 0.01419 & 0.54296 & 0.88215 \\
\hline $\mathrm{C} 17$ & $\mathrm{C}$ & 0.116 & 0.56849 & 0.54169 \\
\hline $\mathrm{C} 18$ & $\mathrm{C}$ & 0.82371 & 0.53587 & 0.49638 \\
\hline
\end{tabular}




\begin{tabular}{|c|c|c|c|c|}
\hline C19 & $\mathrm{C}$ & 0.7448 & 0.56991 & 0.38259 \\
\hline $\mathrm{C} 20$ & $\mathrm{C}$ & 0.78227 & 0.54373 & 0.40786 \\
\hline $\mathrm{C} 21$ & $\mathrm{C}$ & 0.72455 & 0.59904 & 1.04824 \\
\hline N22 & $\mathrm{N}$ & 0.1278 & 0.4211 & 0.80536 \\
\hline $\mathrm{C} 23$ & $\mathrm{C}$ & 0.08284 & 0.54134 & 0.89099 \\
\hline $\mathrm{C} 24$ & $\mathrm{C}$ & -0.09115 & 0.52231 & 0.83646 \\
\hline $\mathrm{C} 25$ & $\mathrm{C}$ & 0.93446 & 0.5131 & 0.53114 \\
\hline $\mathrm{C} 26$ & $\mathrm{C}$ & 0.53956 & 0.59669 & 0.57647 \\
\hline $\mathrm{C} 27$ & $\mathrm{C}$ & 0.51099 & 0.56973 & 0.58322 \\
\hline $\mathrm{C} 28$ & $\mathrm{C}$ & 0.50841 & 0.54535 & 0.53353 \\
\hline $\mathrm{C} 29$ & $\mathrm{C}$ & 0.53278 & 0.54822 & 0.4767 \\
\hline $\mathrm{C} 30$ & $\mathrm{C}$ & 0.56104 & 0.57512 & 0.47021 \\
\hline C31 & $\mathrm{C}$ & 0.56444 & 0.59933 & 0.52008 \\
\hline C 32 & $\mathrm{C}$ & 0.91483 & 0.56477 & 0.4874 \\
\hline C33 & $\mathrm{C}$ & 0.7599 & 0.75753 & 0.77185 \\
\hline C34 & $\mathrm{C}$ & 0.62205 & 0.59308 & 0.804 \\
\hline C35 & $\mathrm{C}$ & 0.58857 & 0.56925 & 0.75443 \\
\hline C36 & $\mathrm{C}$ & 0.62136 & 0.54683 & 0.73435 \\
\hline C37 & $\mathrm{C}$ & 0.68633 & 0.54851 & 0.76185 \\
\hline C38 & $\mathrm{C}$ & 0.72016 & 0.57212 & 0.81204 \\
\hline C39 & $\mathrm{C}$ & 0.68743 & 0.59441 & 0.83294 \\
\hline $\mathrm{C} 40$ & $\mathrm{C}$ & 0.73643 & 0.85769 & 0.87655 \\
\hline C41 & $\mathrm{C}$ & 0.67945 & 0.87265 & 0.83432 \\
\hline $\mathrm{C} 42$ & $\mathrm{C}$ & 0.62358 & 0.85598 & 0.79979 \\
\hline $\mathrm{C} 43$ & $\mathrm{C}$ & 0.62448 & 0.82498 & 0.8127 \\
\hline $\mathrm{C} 44$ & $\mathrm{C}$ & 0.68112 & 0.81062 & 0.85989 \\
\hline $\mathrm{C} 45$ & $\mathrm{C}$ & 0.73726 & 0.82685 & 0.88927 \\
\hline $\mathrm{C} 46$ & $\mathrm{C}$ & 1.02064 & 0.54468 & 0.53776 \\
\hline $\mathrm{C} 47$ & $\mathrm{C}$ & 0.26475 & 0.54032 & 0.27854 \\
\hline N48 & $\mathrm{N}$ & 0.24602 & 0.5616 & 1.22534 \\
\hline C49 & $\mathrm{C}$ & 0.66107 & 0.66525 & 0.73432 \\
\hline C50 & $\mathrm{C}$ & 0.65633 & 0.68869 & 0.78126 \\
\hline
\end{tabular}




\begin{tabular}{|c|c|c|c|c|}
\hline C51 & $\mathrm{C}$ & 0.67937 & 0.837 & 1.10095 \\
\hline C52 & $\mathrm{C}$ & 0.33929 & 0.59945 & 0.86494 \\
\hline C53 & $\mathrm{C}$ & 0.34309 & 0.57556 & 0.91592 \\
\hline C54 & $\mathrm{C}$ & 0.37236 & 0.54874 & 0.91122 \\
\hline C55 & $\mathrm{C}$ & 0.39743 & 0.54563 & 0.85514 \\
\hline C56 & $\mathrm{C}$ & 0.39452 & 0.56971 & 0.80443 \\
\hline C57 & $\mathrm{C}$ & 0.36484 & 0.59657 & 0.80932 \\
\hline C58 & $\mathrm{C}$ & 0.78235 & 0.44659 & 0.56185 \\
\hline C59 & $\mathrm{C}$ & 0.74195 & 0.45978 & 0.48948 \\
\hline C60 & $\mathrm{C}$ & 0.75353 & 0.4884 & 0.46897 \\
\hline C61 & $\mathrm{C}$ & 0.80703 & 0.50464 & 0.51956 \\
\hline C62 & $\mathrm{C}$ & 0.84241 & 0.49276 & 0.59674 \\
\hline C63 & $\mathrm{C}$ & 0.8318 & 0.46354 & 0.61626 \\
\hline C64 & $\mathrm{C}$ & -0.07236 & 0.57371 & 0.88251 \\
\hline C65 & $\mathrm{C}$ & 0.31096 & 0.52574 & 0.42094 \\
\hline C66 & $\mathrm{C}$ & 0.30629 & 0.54686 & 0.36244 \\
\hline C67 & $\mathrm{C}$ & 0.33812 & 0.57445 & 0.38571 \\
\hline C68 & $\mathrm{C}$ & 0.37249 & 0.58105 & 0.4665 \\
\hline C69 & $\mathrm{C}$ & 0.37651 & 0.55995 & 0.52492 \\
\hline C70 & $\mathrm{C}$ & 0.34574 & 0.53221 & 0.50148 \\
\hline C71 & $\mathrm{C}$ & 0.67912 & 0.74217 & 1.07286 \\
\hline N72 & $\mathrm{N}$ & 1.08702 & 0.54766 & 0.56242 \\
\hline C73 & $\mathrm{C}$ & 0.71998 & 0.65343 & 0.74806 \\
\hline N74 & $\mathrm{N}$ & -0.18068 & 0.55221 & 0.82575 \\
\hline C75 & $\mathrm{C}$ & 0.76936 & 0.68843 & 0.85723 \\
\hline C76 & $\mathrm{C}$ & 0.7105 & 0.70053 & 0.84202 \\
\hline C77 & $\mathrm{C}$ & 0.61559 & 0.845 & 1.07059 \\
\hline C78 & $\mathrm{C}$ & 0.07478 & 0.46975 & 0.76845 \\
\hline C79 & $\mathrm{C}$ & 0.06415 & 0.49876 & 0.78926 \\
\hline $\mathrm{C} 80$ & $\mathrm{C}$ & 0.09923 & 0.51017 & 0.86703 \\
\hline C81 & $\mathrm{C}$ & 0.15187 & 0.49343 & 0.91769 \\
\hline C82 & $\mathrm{C}$ & 0.16289 & 0.46481 & 0.89648 \\
\hline
\end{tabular}




\begin{tabular}{|c|c|c|c|c|}
\hline $\mathrm{C} 83$ & $\mathrm{C}$ & 0.12331 & 0.45225 & 0.82267 \\
\hline $\mathrm{C} 84$ & $\mathrm{C}$ & 0.04468 & 0.57862 & 0.77256 \\
\hline $\mathrm{C} 85$ & $\mathrm{C}$ & 0.09405 & 0.5632 & 0.83381 \\
\hline $\mathrm{C} 86$ & $\mathrm{C}$ & 0.15468 & 0.56526 & 0.83576 \\
\hline $\mathrm{C} 87$ & $\mathrm{C}$ & 0.16603 & 0.58356 & 0.78226 \\
\hline $\mathrm{C} 88$ & $\mathrm{C}$ & 0.11743 & 0.59995 & 0.72501 \\
\hline $\mathrm{C} 89$ & $\mathrm{C}$ & 0.05634 & 0.59719 & 0.71996 \\
\hline C90 & $\mathrm{C}$ & 0.78404 & 0.52449 & 0.34845 \\
\hline C91 & $\mathrm{C}$ & 0.89224 & 0.53749 & 0.50493 \\
\hline C92 & $\mathrm{C}$ & 0.77415 & 0.66483 & 0.81067 \\
\hline C93 & $\mathrm{C}$ & 0.69892 & 0.81473 & 1.06365 \\
\hline C94 & $\mathrm{C}$ & 0.61977 & 0.74316 & 1.07403 \\
\hline C95 & $\mathrm{C}$ & 0.81292 & 0.55759 & 0.55418 \\
\hline C96 & $\mathrm{C}$ & 0.86226 & 0.57322 & 0.61509 \\
\hline C97 & $\mathrm{C}$ & 0.84995 & 0.59265 & 0.66573 \\
\hline C98 & $\mathrm{C}$ & 0.78889 & 0.5964 & 0.65843 \\
\hline C99 & $\mathrm{C}$ & 0.74041 & 0.57869 & 0.60382 \\
\hline C100 & $\mathrm{C}$ & 0.75236 & 0.55959 & 0.55234 \\
\hline C101 & $\mathrm{C}$ & 0.59325 & 0.86667 & 1.11214 \\
\hline $\mathrm{C} 102$ & $\mathrm{C}$ & 0.16225 & 0.57468 & 1.00603 \\
\hline C103 & $\mathrm{C}$ & 0.12403 & 0.54879 & 0.9797 \\
\hline C104 & $\mathrm{C}$ & 0.12161 & 0.52929 & 1.03822 \\
\hline C105 & $\mathrm{C}$ & 0.15946 & 0.5337 & 1.11891 \\
\hline C106 & $\mathrm{C}$ & 0.2023 & 0.55767 & 1.14358 \\
\hline C107 & $\mathrm{C}$ & 0.20154 & 0.57871 & 1.08691 \\
\hline C108 & $\mathrm{C}$ & 0.59656 & 0.52479 & 0.96421 \\
\hline C109 & $\mathrm{C}$ & 0.56115 & 0.53186 & 0.8842 \\
\hline C110 & $\mathrm{C}$ & 0.52945 & 0.55946 & 0.86266 \\
\hline C111 & $\mathrm{C}$ & 0.53317 & 0.5798 & 0.92258 \\
\hline C112 & $\mathrm{C}$ & 0.56797 & 0.57252 & 1.0029 \\
\hline C113 & $\mathrm{C}$ & 0.60087 & 0.54512 & 1.02421 \\
\hline C114 & $\mathrm{C}$ & 0.67442 & 0.77522 & 0.96016 \\
\hline
\end{tabular}




\begin{tabular}{|c|c|c|c|c|}
\hline C115 & $\mathrm{C}$ & 0.68552 & 0.74679 & 0.99706 \\
\hline C116 & $\mathrm{C}$ & 0.70177 & 0.72259 & 0.96073 \\
\hline C117 & $\mathrm{C}$ & 0.70501 & 0.7267 & 0.88696 \\
\hline C118 & $\mathrm{C}$ & 0.69636 & 0.75541 & 0.85176 \\
\hline C119 & $\mathrm{C}$ & 0.68172 & 0.77974 & 0.8891 \\
\hline C120 & $\mathrm{C}$ & 0.57216 & 0.83112 & 1.00148 \\
\hline C121 & $\mathrm{C}$ & 0.59177 & 0.80878 & 0.96443 \\
\hline C122 & $\mathrm{C}$ & 0.77502 & 0.64936 & 1.0581 \\
\hline C123 & $\mathrm{C}$ & 0.7689 & 0.67948 & 1.03475 \\
\hline C124 & $\mathrm{C}$ & 0.70915 & 0.69223 & 0.99544 \\
\hline C125 & $\mathrm{C}$ & 0.65576 & 0.67541 & 0.98408 \\
\hline C126 & $\mathrm{C}$ & 0.66179 & 0.6454 & 1.00736 \\
\hline C127 & $\mathrm{C}$ & 0.7212 & 0.6319 & 1.04213 \\
\hline C128 & $\mathrm{C}$ & 0.70465 & 0.55391 & 0.24415 \\
\hline C129 & $\mathrm{C}$ & 0.64264 & 0.53787 & 1.10764 \\
\hline C130 & $\mathrm{C}$ & 0.70583 & 0.57457 & 0.30185 \\
\hline C131 & $\mathrm{C}$ & 0.67914 & 0.90557 & 0.83814 \\
\hline C132 & $\mathrm{C}$ & 0.655 & 0.80034 & 0.99606 \\
\hline C133 & $\mathrm{C}$ & 0.64769 & 0.76602 & 0.70534 \\
\hline C134 & $\mathrm{C}$ & 0.74667 & 0.52959 & 0.26786 \\
\hline C135 & $\mathrm{C}$ & 0.72618 & 0.73158 & 1.21483 \\
\hline C136 & $\mathrm{C}$ & 0.73235 & 0.73631 & 1.14369 \\
\hline C137 & $\mathrm{C}$ & 0.71067 & 0.76755 & 0.63 \\
\hline C138 & $\mathrm{C}$ & 0.70147 & 0.75984 & 0.77519 \\
\hline C139 & $\mathrm{C}$ & 0.65237 & 0.76992 & 0.63303 \\
\hline C140 & $\mathrm{C}$ & 0.76438 & 0.76137 & 0.69938 \\
\hline N141 & $\mathrm{N}$ & 0.66127 & 0.55857 & 0.16246 \\
\hline N142 & $\mathrm{N}$ & 0.77764 & 0.41519 & 0.5761 \\
\hline N143 & $\mathrm{N}$ & 0.13278 & 0.62017 & 0.67591 \\
\hline C144 & $\mathrm{C}$ & -0.00888 & 0.57028 & 0.89883 \\
\hline C145 & $\mathrm{C}$ & 0.72414 & 0.62981 & 0.6955 \\
\hline N146 & $\mathrm{N}$ & 0.77819 & 0.61871 & 0.70689 \\
\hline
\end{tabular}




\begin{tabular}{|c|c|c|c|c|}
\hline C147 & $\mathrm{C}$ & 0.61386 & 0.73856 & 1.14536 \\
\hline C148 & $\mathrm{C}$ & 0.667 & 0.73274 & 1.21571 \\
\hline C149 & $\mathrm{C}$ & 0.99802 & 0.51728 & 0.55176 \\
\hline $\mathrm{C} 150$ & $\mathrm{C}$ & 0.97811 & 0.56831 & 0.50313 \\
\hline H151 & $\mathrm{H}$ & 0.81394 & 0.59202 & 0.8813 \\
\hline H152 & $\mathrm{H}$ & -0.01269 & 0.49605 & 0.84992 \\
\hline H153 & $\mathrm{H}$ & 0.19035 & 0.6093 & 0.51381 \\
\hline H154 & $\mathrm{H}$ & 0.19773 & 0.52761 & 0.64349 \\
\hline H155 & $\mathrm{H}$ & 0.31154 & 0.52646 & 0.6919 \\
\hline H156 & $\mathrm{H}$ & 0.30539 & 0.60908 & 0.56654 \\
\hline H157 & $\mathrm{H}$ & 0.09023 & 0.58583 & 0.49965 \\
\hline H158 & $\mathrm{H}$ & 0.74481 & 0.5869 & 0.42433 \\
\hline H159 & $\mathrm{H}$ & 0.68135 & 0.58685 & 1.0187 \\
\hline H160 & $\mathrm{H}$ & -0.12243 & 0.50367 & 0.80962 \\
\hline H161 & $\mathrm{H}$ & 0.91997 & 0.49071 & 0.53817 \\
\hline H162 & $\mathrm{H}$ & 0.54165 & 0.61571 & 0.61406 \\
\hline H163 & $\mathrm{H}$ & 0.48657 & 0.52438 & 0.5379 \\
\hline H164 & $\mathrm{H}$ & 0.52905 & 0.52973 & 0.43699 \\
\hline H165 & $\mathrm{H}$ & 0.58006 & 0.57725 & 0.42632 \\
\hline H166 & $\mathrm{H}$ & 0.58601 & 0.62022 & 0.51466 \\
\hline H167 & $\mathrm{H}$ & 0.8837 & 0.58385 & 0.46473 \\
\hline H168 & $\mathrm{H}$ & 0.8018 & 0.75276 & 0.82526 \\
\hline H169 & $\mathrm{H}$ & 0.59752 & 0.61062 & 0.82016 \\
\hline $\mathrm{H} 170$ & $\mathrm{H}$ & 0.59656 & 0.52807 & 0.69706 \\
\hline H171 & $\mathrm{H}$ & 0.71002 & 0.53128 & 0.74379 \\
\hline H172 & $\mathrm{H}$ & 0.71243 & 0.61278 & 0.87178 \\
\hline H173 & $\mathrm{H}$ & 0.77992 & 0.8701 & 0.90408 \\
\hline H174 & $\mathrm{H}$ & 0.57901 & 0.86708 & 0.76719 \\
\hline $\mathrm{H} 175$ & $\mathrm{H}$ & 0.58081 & 0.81254 & 0.78999 \\
\hline H176 & $\mathrm{H}$ & 0.78115 & 0.81591 & 0.92616 \\
\hline H177 & $\mathrm{H}$ & 0.24856 & 0.51742 & 0.2629 \\
\hline H178 & $\mathrm{H}$ & 0.61889 & 0.6566 & 0.68668 \\
\hline
\end{tabular}




\begin{tabular}{|c|c|c|c|c|}
\hline H179 & $\mathrm{H}$ & 0.61068 & 0.69804 & 0.76947 \\
\hline H180 & $\mathrm{H}$ & 0.71387 & 0.84753 & 1.15411 \\
\hline H181 & $\mathrm{H}$ & 0.31658 & 0.62021 & 0.86858 \\
\hline H182 & $\mathrm{H}$ & 0.32344 & 0.57786 & 0.95912 \\
\hline H183 & $\mathrm{H}$ & 0.37619 & 0.53047 & 0.95159 \\
\hline H184 & $\mathrm{H}$ & 0.41994 & 0.5247 & 0.85205 \\
\hline H185 & $\mathrm{H}$ & 0.36217 & 0.61533 & 0.77066 \\
\hline H186 & $\mathrm{H}$ & 0.7024 & 0.44762 & 0.44672 \\
\hline H187 & $\mathrm{H}$ & 0.72003 & 0.49735 & 0.41293 \\
\hline H188 & $\mathrm{H}$ & 0.88097 & 0.50549 & 0.64017 \\
\hline H189 & $\mathrm{H}$ & 0.86274 & 0.45396 & 0.67311 \\
\hline H190 & $\mathrm{H}$ & -0.08837 & 0.59542 & 0.89276 \\
\hline H191 & $\mathrm{H}$ & 0.28615 & 0.50451 & 0.40435 \\
\hline H192 & $\mathrm{H}$ & 0.3352 & 0.59116 & 0.34167 \\
\hline H193 & $\mathrm{H}$ & 0.39604 & 0.60255 & 0.48357 \\
\hline H194 & $\mathrm{H}$ & 0.34875 & 0.51557 & 0.54574 \\
\hline H195 & $\mathrm{H}$ & 0.8114 & 0.69765 & 0.90398 \\
\hline H196 & $\mathrm{H}$ & 0.04439 & 0.46069 & 0.71066 \\
\hline H197 & $\mathrm{H}$ & 0.02596 & 0.51175 & 0.7461 \\
\hline H198 & $\mathrm{H}$ & 0.18506 & 0.50191 & 0.97449 \\
\hline H199 & $\mathrm{H}$ & 0.20147 & 0.45235 & 0.93988 \\
\hline $\mathrm{H} 200$ & $\mathrm{H}$ & -0.00356 & 0.57634 & 0.76347 \\
\hline H201 & $\mathrm{H}$ & 0.1934 & 0.55266 & 0.87927 \\
\hline H202 & $\mathrm{H}$ & 0.21291 & 0.58503 & 0.78523 \\
\hline H203 & $\mathrm{H}$ & 0.01718 & 0.60937 & 0.67642 \\
\hline H204 & $\mathrm{H}$ & 0.81304 & 0.50468 & 0.3659 \\
\hline H205 & $\mathrm{H}$ & 0.82018 & 0.65593 & 0.82273 \\
\hline H206 & $\mathrm{H}$ & 0.74788 & 0.80823 & 1.08818 \\
\hline H207 & $\mathrm{H}$ & 0.5782 & 0.74736 & 1.01972 \\
\hline H208 & $\mathrm{H}$ & 0.91063 & 0.57062 & 0.62487 \\
\hline H209 & $\mathrm{H}$ & 0.88811 & 0.60522 & 0.71025 \\
\hline $\mathrm{H} 210$ & $\mathrm{H}$ & 0.69342 & 0.57934 & 0.60044 \\
\hline
\end{tabular}




\begin{tabular}{|c|c|c|c|c|}
\hline H211 & $\mathrm{H}$ & 0.71392 & 0.5465 & 0.50993 \\
\hline $\mathrm{H} 212$ & $\mathrm{H}$ & 0.54353 & 0.87112 & 1.08874 \\
\hline $\mathrm{H} 213$ & $\mathrm{H}$ & 0.16272 & 0.59187 & 0.96502 \\
\hline $\mathrm{H} 214$ & $\mathrm{H}$ & 0.09201 & 0.5097 & 1.01991 \\
\hline $\mathrm{H} 215$ & $\mathrm{H}$ & 0.15508 & 0.51824 & 1.16139 \\
\hline $\mathrm{H} 216$ & $\mathrm{H}$ & 0.23219 & 0.59811 & 1.10539 \\
\hline $\mathrm{H} 217$ & $\mathrm{H}$ & 0.622 & 0.5037 & 0.97923 \\
\hline H218 & $\mathrm{H}$ & 0.55842 & 0.51578 & 0.83883 \\
\hline H219 & $\mathrm{H}$ & 0.50896 & 0.60119 & 0.90707 \\
\hline $\mathrm{H} 220$ & $\mathrm{H}$ & 0.57048 & 0.58859 & 1.04814 \\
\hline $\mathrm{H} 221$ & $\mathrm{H}$ & 0.52291 & 0.83709 & 0.9773 \\
\hline $\mathrm{H} 222$ & $\mathrm{H}$ & 0.55761 & 0.79754 & 0.91228 \\
\hline $\mathrm{H} 223$ & $\mathrm{H}$ & 0.82134 & 0.63938 & 1.08432 \\
\hline $\mathrm{H} 224$ & $\mathrm{H}$ & 0.81042 & 0.6925 & 1.04419 \\
\hline $\mathrm{H} 225$ & $\mathrm{H}$ & 0.60944 & 0.68519 & 0.95406 \\
\hline H226 & $\mathrm{H}$ & 0.61975 & 0.6323 & 0.99423 \\
\hline $\mathrm{H} 227$ & $\mathrm{H}$ & 0.65876 & 0.51489 & 1.12158 \\
\hline H228 & $\mathrm{H}$ & 0.67586 & 0.59423 & 0.28424 \\
\hline H229 & $\mathrm{H}$ & 0.72229 & 0.91629 & 0.87676 \\
\hline $\mathrm{H} 230$ & $\mathrm{H}$ & 0.60223 & 0.76761 & 0.70692 \\
\hline H231 & $\mathrm{H}$ & 0.75087 & 0.51441 & 0.2247 \\
\hline $\mathrm{H} 232$ & $\mathrm{H}$ & 0.76727 & 0.727 & 1.26927 \\
\hline $\mathrm{H} 233$ & $\mathrm{H}$ & 0.77844 & 0.73539 & 1.14371 \\
\hline $\mathrm{H} 234$ & $\mathrm{H}$ & 0.71421 & 0.77051 & 0.57393 \\
\hline $\mathrm{H} 235$ & $\mathrm{H}$ & 0.61079 & 0.77468 & 0.57932 \\
\hline H236 & $\mathrm{H}$ & 0.80948 & 0.75957 & 0.69699 \\
\hline $\mathrm{H} 237$ & $\mathrm{H}$ & 0.02198 & 0.5895 & 0.92086 \\
\hline H238 & $\mathrm{H}$ & 0.68168 & 0.62284 & 0.64577 \\
\hline H239 & $\mathrm{H}$ & 0.56805 & 0.7394 & 1.14603 \\
\hline $\mathrm{H} 240$ & $\mathrm{H}$ & 0.66231 & 0.72906 & 1.27085 \\
\hline $\mathrm{H} 241$ & $\mathrm{H}$ & 1.02971 & 0.49889 & 0.57966 \\
\hline H242 & $\mathrm{H}$ & 0.99347 & 0.58987 & 0.49113 \\
\hline
\end{tabular}




\section{Section S5. The Framework Energy Calculation}

Starting from TAPM and TPB-Ph, we also built a 3D structure with non-interpenetrated pts topology. We then compared the framework Energies between this model and 3DTPB-COF-Ph (ljh topology) via the Forcite modules in Materials Studio v7.0 ${ }^{\mathrm{S} 11}$. The force field of COMPASS II was used with parameters of c3=, c40 (only for -CH3 group), $\mathrm{n} 2=$ and $\mathrm{h}$ for carbon, nitrogen and hydrogen, respectively. The Smart algorithm was used to optimize the geometry and unit cell parameters with ultrafine quality. As shown in table $\mathrm{S} 4$, the energy of 3D-TPB-COF-Ph is much lower.

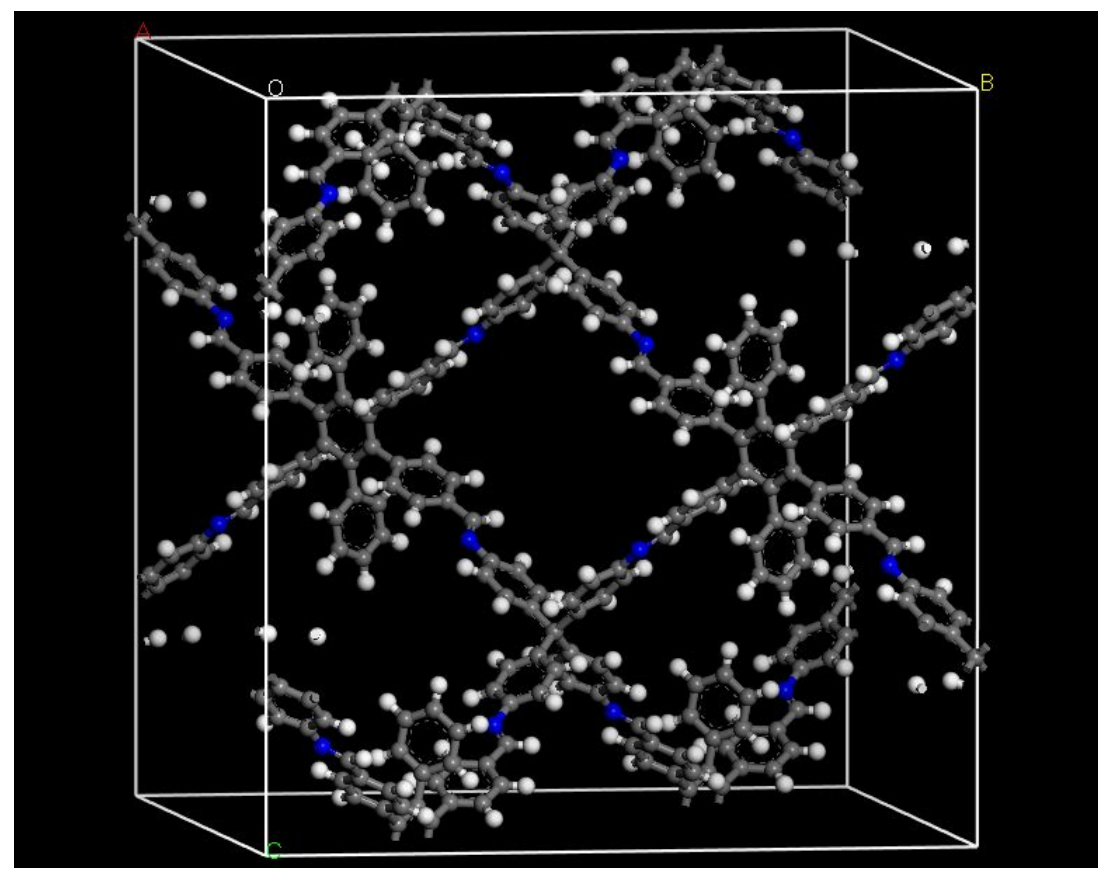

Figure S13 View of non-interpenetrated pts topology starting from TAPM and TPB$\mathrm{Ph}$.

Table S5 The energies for different 3D frameworks.

\begin{tabular}{|c|c|c|}
\hline Topology & Formula & Framework energy (Kcal/mol) \\
\hline pts (non-interpenetrated) & $\mathrm{C}_{71} \mathrm{H}_{46} \mathrm{~N}_{4}$ & 1803.23 \\
\hline $\mathbf{l j h}$ & $\mathrm{C}_{71} \mathrm{H}_{46} \mathrm{~N}_{4}$ & 1495.04 \\
\hline
\end{tabular}




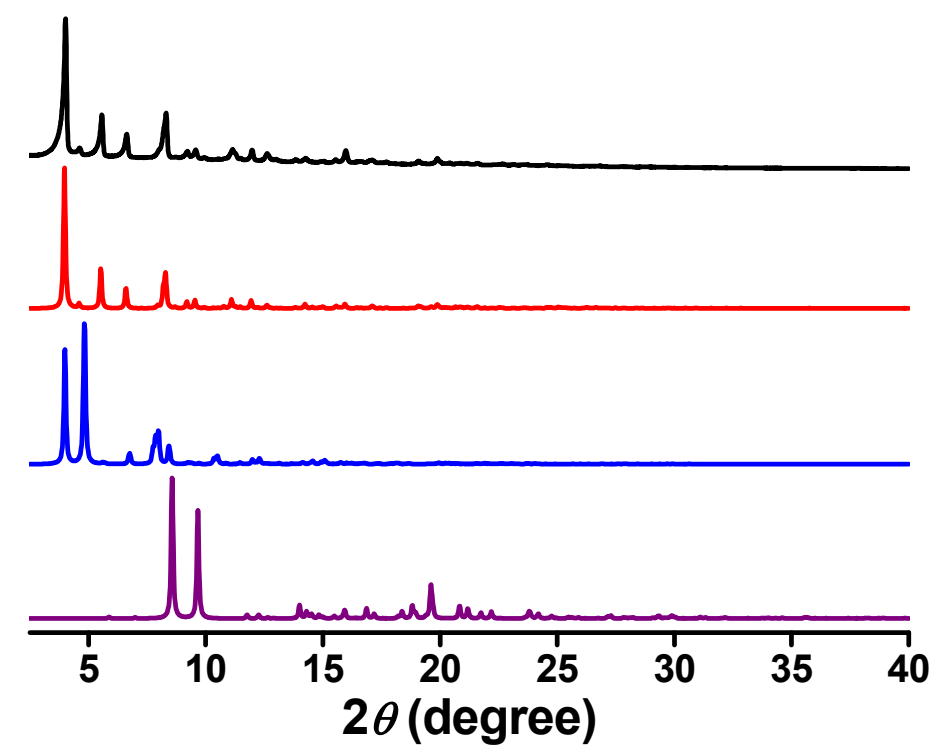

Figure S14 The experimental (black curve) and calculated (red curve) PXRD patterns of 3D-TPB-COF-Ph, the calculated PXRD pattern of non-interpenetrated pts topology starting from TAPM and TPB-Ph (blue curve), and the calculated PXRD pattern of 3DTPB-COF-OMe with five-interpenetrated pts structure (purple curve).

\section{Section S6. NMR Spectra}



Figure $\mathbf{S 1 5}{ }^{1} \mathrm{H}$ NMR spectrum $\left(\mathrm{CDCl}_{3}, 400 \mathrm{MHz}\right)$ of compound $\mathbf{1}$ 


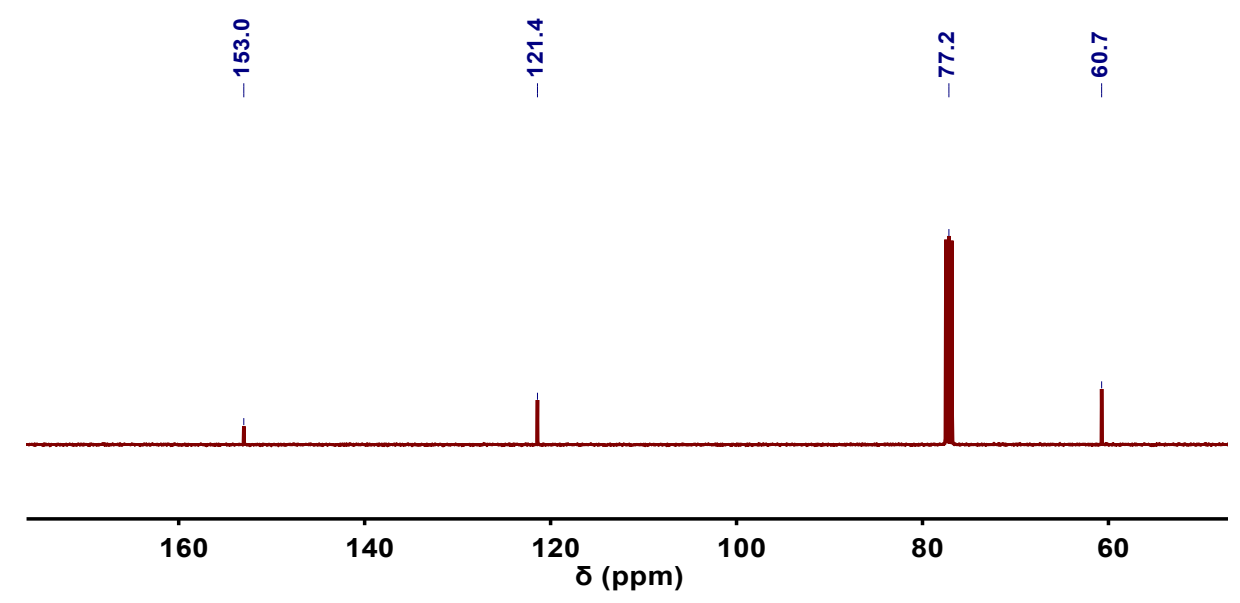

Figure S16 ${ }^{13} \mathrm{C}$ NMR spectrum $\left(\mathrm{CDCl}_{3}, 100 \mathrm{MHz}\right)$ of compound $\mathbf{1}$

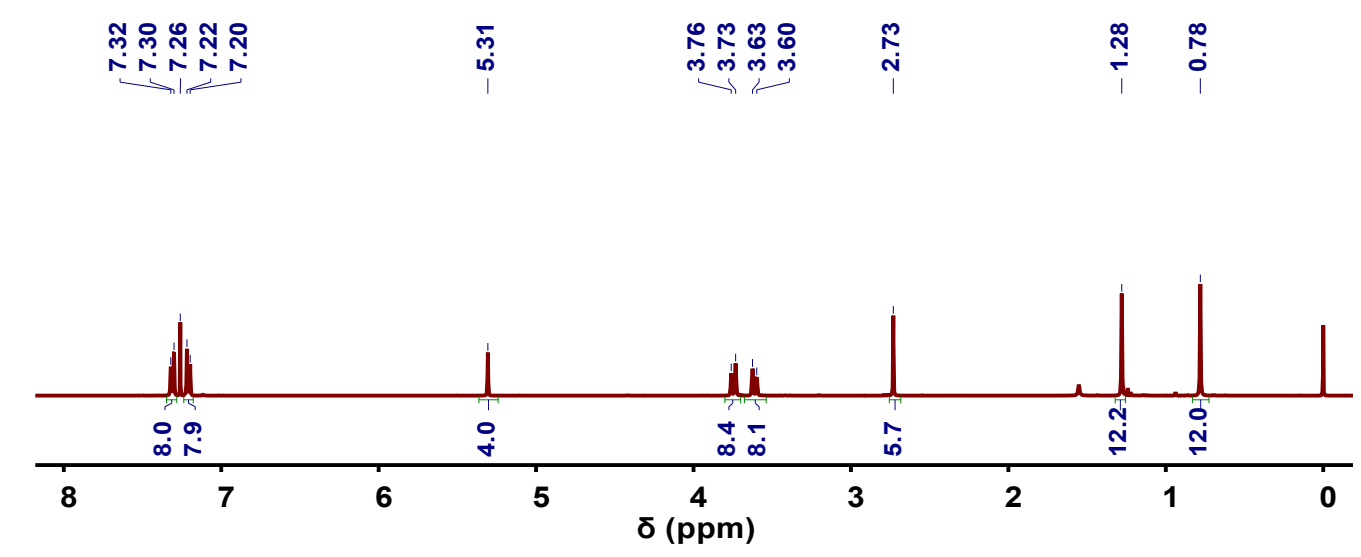

Figure $\mathbf{S 1 7}{ }^{1} \mathrm{H}$ NMR spectrum $\left(\mathrm{CDCl}_{3}, 400 \mathrm{MHz}\right)$ of compound 3

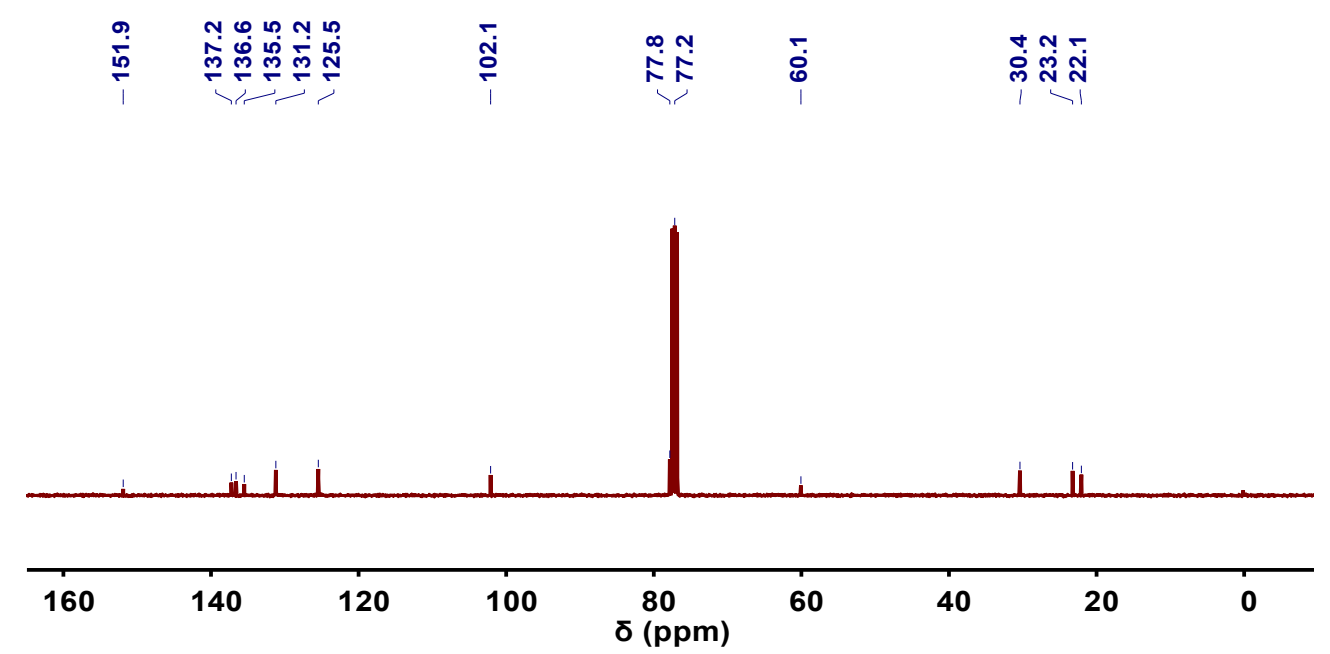

Figure S18 ${ }^{13} \mathrm{C}$ NMR spectrum $\left(\mathrm{CDCl}_{3}, 100 \mathrm{MHz}\right)$ of compound 3 
م:

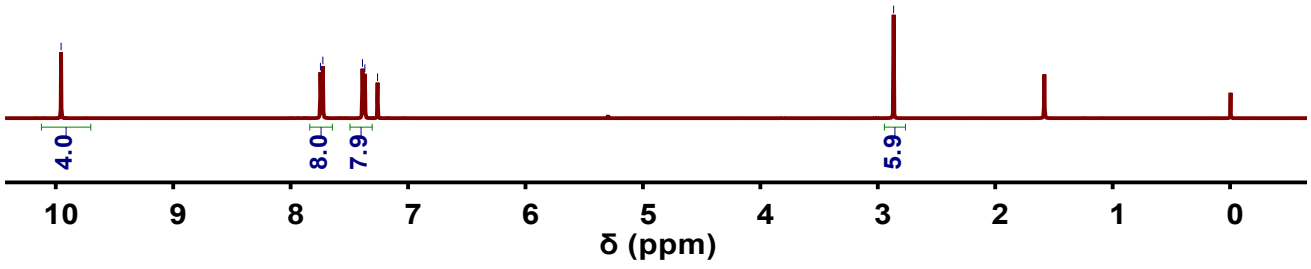

Figure $\mathbf{S 1 9}{ }^{1} \mathrm{H}$ NMR spectrum $\left(\mathrm{CDCl}_{3}, 400 \mathrm{MHz}\right)$ of TPB-OMe
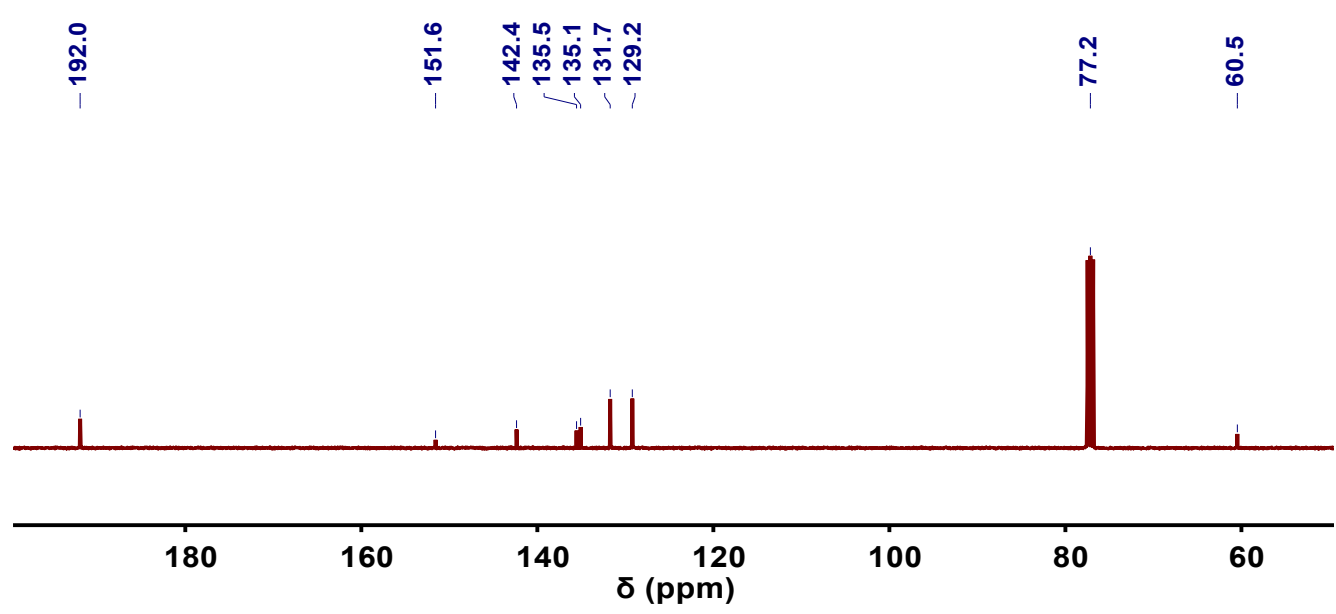

Figure $\mathbf{S 2 0}{ }^{13} \mathrm{C}$ NMR spectrum $\left(\mathrm{CDCl}_{3}, 100 \mathrm{MHz}\right)$ of TPB-OMe.

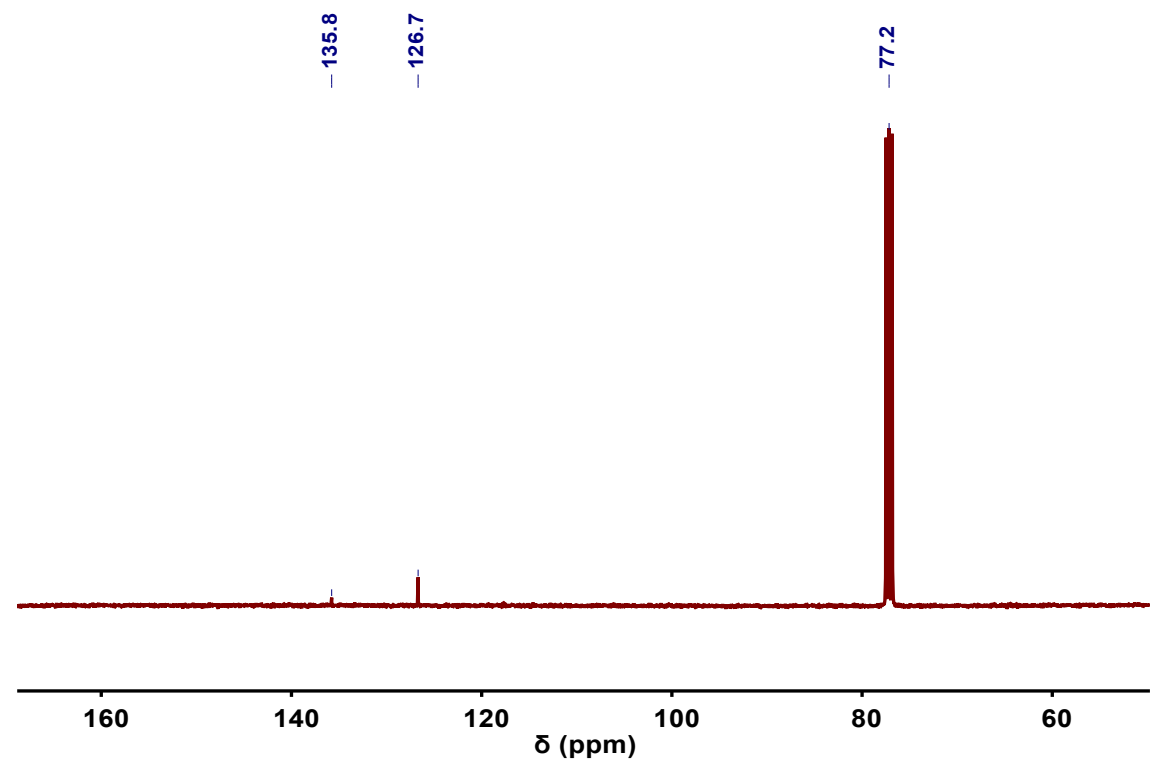

Figure S21 ${ }^{13} \mathrm{C}$ NMR spectrum $\left(\mathrm{CDCl}_{3}, 100 \mathrm{MHz}\right)$ of compound 4 


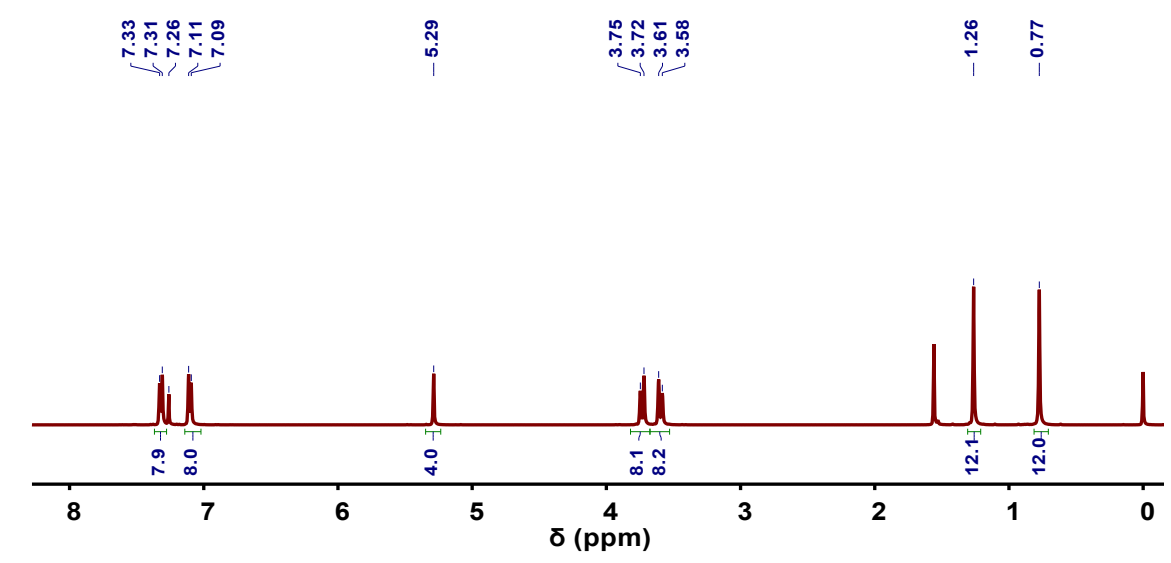

Figure S22 ${ }^{1} \mathrm{H}$ NMR spectrum $\left(\mathrm{CDCl}_{3}, 400 \mathrm{MHz}\right)$ of compound 5

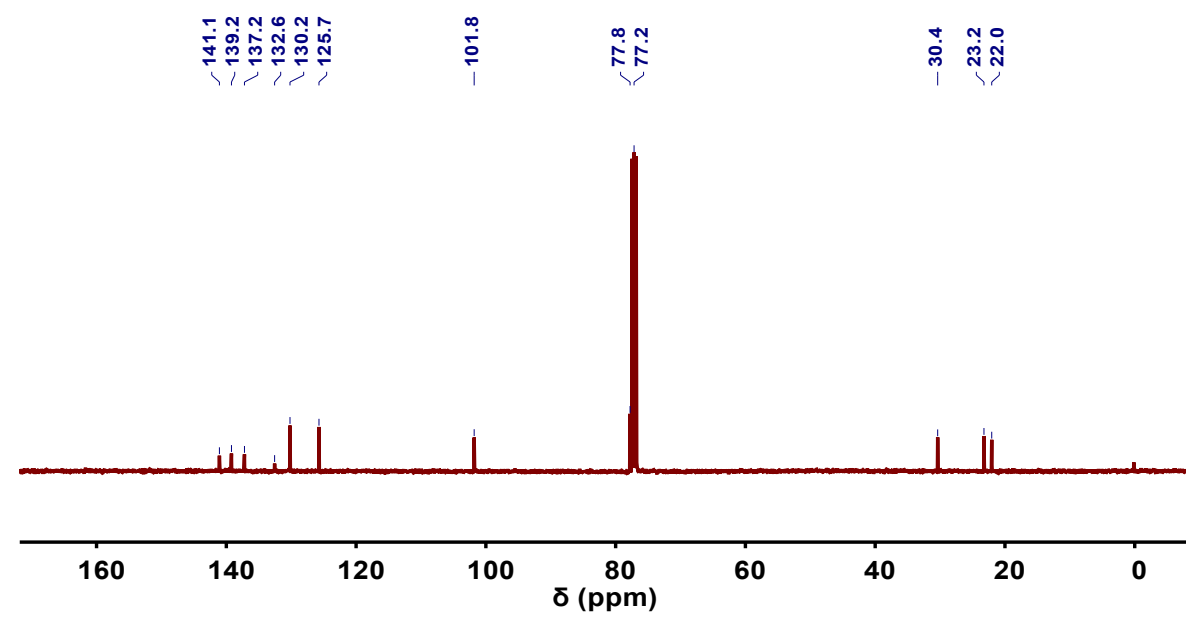

Figure $\mathbf{S 2 3}{ }^{13} \mathrm{C}$ NMR spectrum $\left(\mathrm{CDCl}_{3}, 100 \mathrm{MHz}\right)$ of compound 5

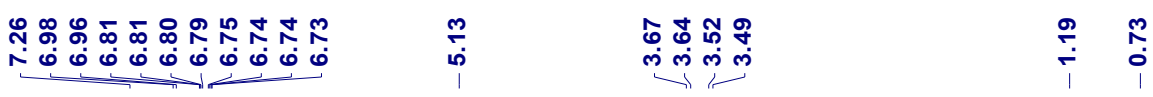

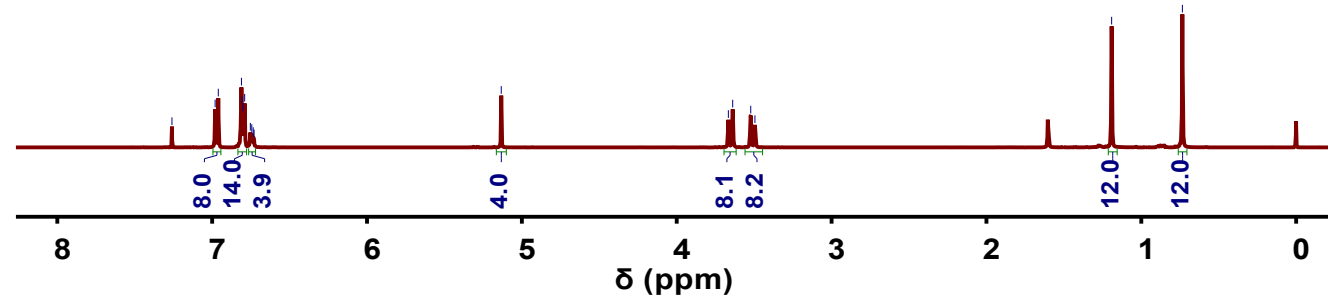

Figure S24 ${ }^{1} \mathrm{H}$ NMR spectrum $\left(\mathrm{CDCl}_{3}, 400 \mathrm{MHz}\right)$ of compound 6 


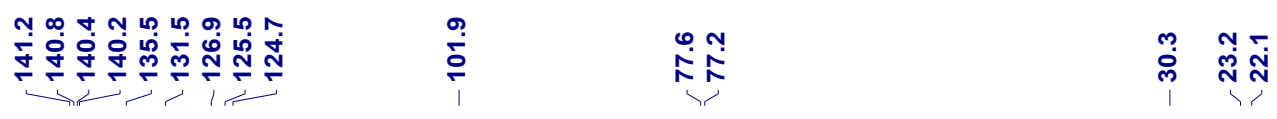

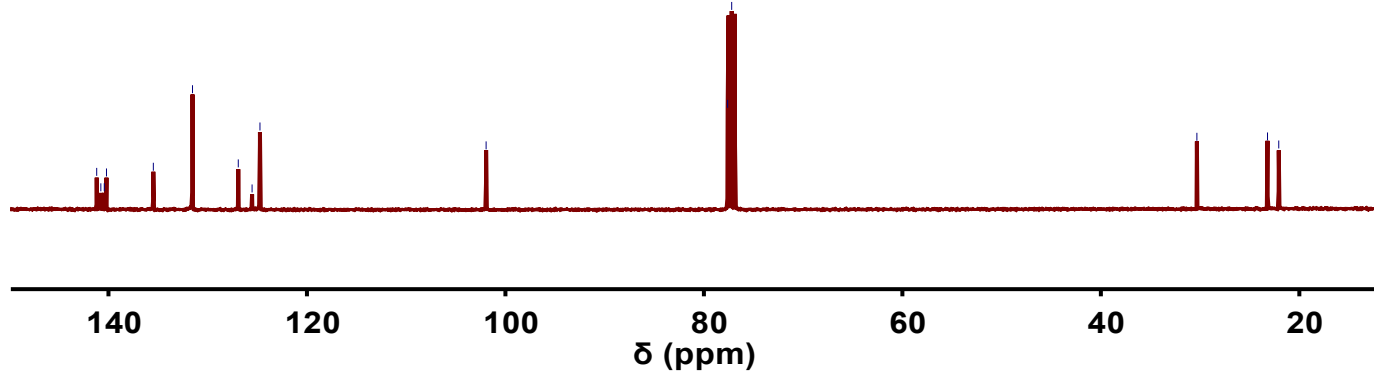

Figure S25 ${ }^{13} \mathrm{C}$ NMR spectrum $\left(\mathrm{CDCl}_{3}, 100 \mathrm{MHz}\right)$ of compound 6

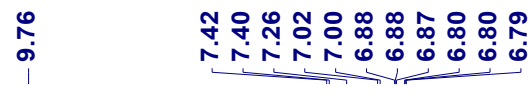

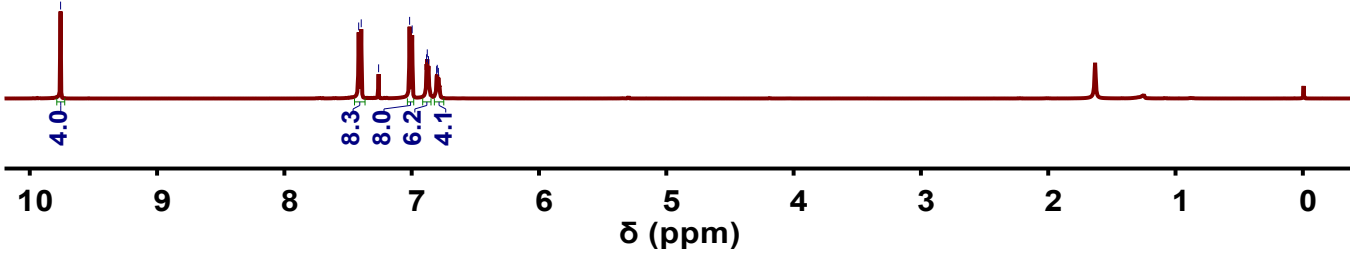

Figure S26 ${ }^{1} \mathrm{H}$ NMR spectrum $\left(\mathrm{CDCl}_{3}, 400 \mathrm{MHz}\right)$ of TPB-Ph

ํำ

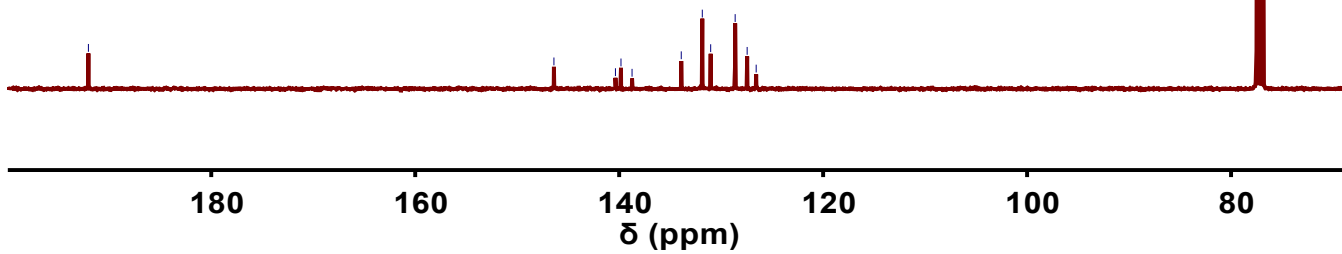

Figure $\mathbf{S 2 7}{ }^{13} \mathrm{C}$ NMR spectrum $\left(\mathrm{CDCl}_{3}, 100 \mathrm{MHz}\right)$ of TPB-Ph 


\section{Section S7. References}

S1 Rack, M.; Hauschel, B.; Hanack, M. Nickel Hemiporphyrazines as Bisdienes and Bisdienophiles: Synthesis and Characterization, Chem. Ber. 1996, 129, 237-242.

S2 Gao, C.; Li, J.; Yin, S.; Lin, G.; Ma, T.; Meng, Y.; Sun, J.; Wang, C. Isostructural Three-Dimensional Covalent Organic Frameworks, Angew. Chem. Int. Ed. 2019, $58,9770-9775$.

S3 Shigehisa, A.; Takahiro, O.; Tatsuya, N. A novel graphite-like stacking structure in a discrete molecule and its molecular recognition behavior, New J. Chem. 2018, 42, 9369-9372.

S4 Ganesan, P.; Yang, X. N.; Loos, J.; Savenije, T. J.; Abellon, R. D.; Zuilhof, H.; Sudholter, E. J. R. Tetrahedral $n$-type materials: Efficient quenching of the excitation of $p$-type polymers in amorphous films. J. Am. Chem. Soc. 2005, 127, 14530.

S5 Gao, C.; Li, J.; Yin, S.; Sun, J.; Wang, C. Twist Building Blocks from Planar to Tetrahedral for the Synthesis of Covalent Organic Frameworks, J. Am. Chem. Soc. 2020, 142, 3718-3723.

S6 Smeets, S., Wang, B., Cichocka, M. O., Angstrom, J.; Wan, W. (2018, April 11) Instamatic (Version 0.6) Zenodo. https://doi.org/10.5281/zenodo.1217026

S7 Kabsch, W. Integration, scaling, space-group assignment and post-refinement. Acta Cryst. 2010, D66, 133-144.

S8 Wan, W.; Sun, J.; Su, J.; Hovmoller, S.; Zou, X. Three-dimensional rotation electron diffraction: software RED for automated data collection and data processing. J. Appl. Cryst. 2013, 46, 1863-1873.

S9 Sheldrick, G. M. SHELXT-Integrated space-group and crystal-structure determination. Acta Cryst. 2015, 71, 3-8.

S10 Coelho, A. A. TOPAS-ACADEMIC v5.0; 2012.

S11 Ccelrys Software Inc., Materials Studio Release Notes, Release 7.0, San Diego: Accelrys Software Inc., 2013. 